PREPARED FOR THE U.S. DEPARTMENT OF ENERGY, UNDER CONTRACT DE-AC02-76CH03073

PPPL-3708

PPPL-3708

UC-70

Experimental Study of Lower-hybrid Drift Turbulence in a Reconnecting Current Sheet

by

T.A. Carter, M. Yamada, H. Ji, R.M. Kulsrud, and F. Trintchouk

June 2002

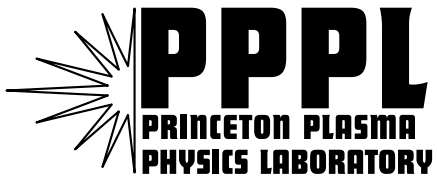

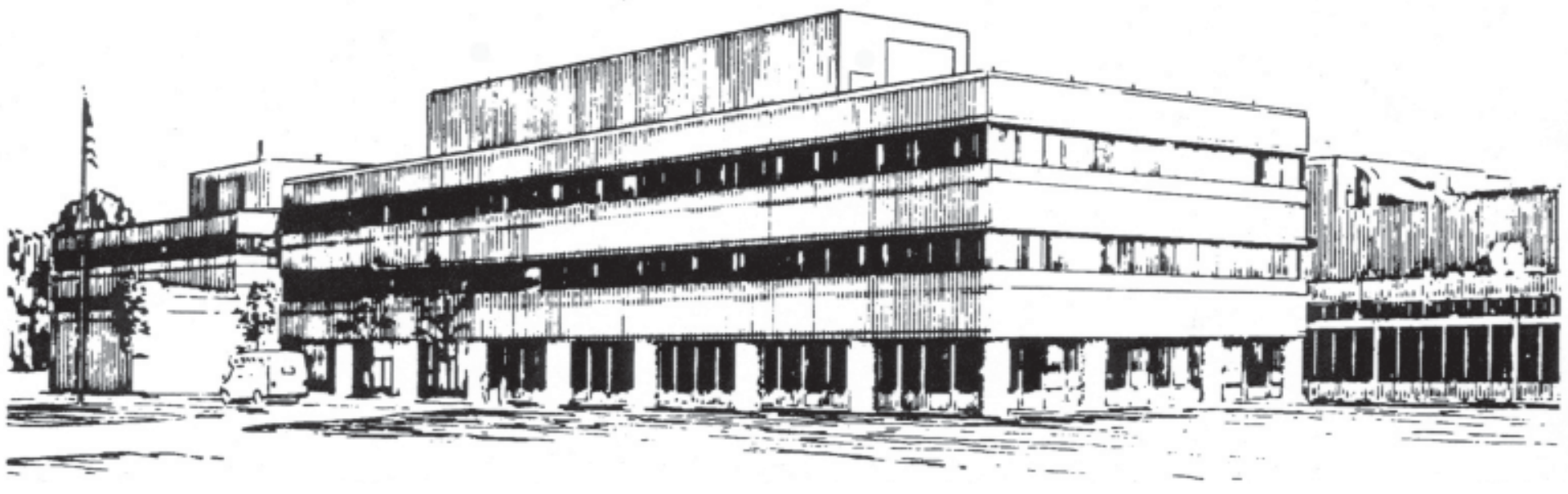

PRINCETON PLASMA PHYSICS LABORATORY PRINCETON UNIVERSITY, PRINCETON, NEW JERSEY 


\section{PPPL Reports Disclaimer}

This report was prepared as an account of work sponsored by an agency of the United States Government. Neither the United States Government nor any agency thereof, nor any of their employees, makes any warranty, express or implied, or assumes any legal liability or responsibility for the accuracy, completeness, or usefulness of any information, apparatus, product, or process disclosed, or represents that its use would not infringe privately owned rights. Reference herein to any specific commercial product, process, or service by trade name, trademark, manufacturer, or otherwise, does not necessarily constitute or imply its endorsement, recommendation, or favoring by the United States Government or any agency thereof. The views and opinions of authors expressed herein do not necessarily state or reflect those of the United States Government or any agency thereof.

\section{Availability}

This report is posted on the U.S. Department of Energy's Princeton Plasma Physics Laboratory Publications and Reports web site in Fiscal Year 2002. The home page for PPPL Reports and Publications is: http://www.pppl.gov/pub_report/

DOE and DOE Contractors can obtain copies of this report from:

U.S. Department of Energy

Office of Scientific and Technical Information

DOE Technical Information Services (DTIS)

P.O. Box 62

Oak Ridge, TN 37831

Telephone: (865) 576-8401

Fax: (865) 576-5728

Email: reports@adonis.osti.gov

This report is available to the general public from:

National Technical Information Service

U.S. Department of Commerce

5285 Port Royal Road

Springfield, VA 22161

Telephone: 1-800-553-6847 or

(703) 605-6000

Fax: (703) 321-8547

Internet: http://www.ntis.gov/ordering.htm 


\title{
Experimental study of lower-hybrid drift turbulence in a reconnecting current sheet
}

\author{
T. A. Carter, ${ }^{*}$ M. Yamada, H. Ji, R. M. Kulsrud, and F. Trintchouk ${ }^{\dagger}$ \\ Princeton University, Plasma Physics Laboratory, \\ P.O. Box 451, Princeton, New Jersey 08543
}

(Dated: June 11, 2002) 


\begin{abstract}
The role of turbulence in the process of magnetic reconnection has been the subject of a great deal of study and debate in the theoretical literature. At issue in this debate is whether turbulence is essential for fast magnetic reconnection to occur in collisionless current sheets. Some theories claim it is necessary in order to provide anomalous resistivity, while others present a laminar fast reconnection mechanism based on the Hall term in the generalized Ohm's law. In this work, a thorough study of electrostatic potential fluctuations in the current sheet of the Magnetic Reconnection Experiment (MRX) [M. Yamada et al., Phys. Plasmas 4, 1936 (1997)] was performed in order to ascertain the importance of turbulence in a laboratory reconnection experiment. Using amplified floating Langmuir probes, broadband fluctuations in the lower hybrid frequency range $\left(f_{\mathrm{LH}} \sim 5-15 \mathrm{MHz}\right)$ were measured which arise with the formation of the current sheet in MRX. The frequency spectrum, spatial amplitude profile, and spatial correlation characteristics of the measured turbulence were examined carefully, finding consistency with theories of the lower-hybrid drift instability (LHDI). The LHDI and its role in magnetic reconnection has been studied theoretically for decades, but this work represents the first detection and detailed study of the LHDI in a laboratory current sheet. The observation of the LHDI in MRX has provided the unique opportunity to uncover the role of this instability in collisionless reconnection. It was found that: (1) the LHDI fluctuations are confined to the low-beta edge of current sheets in MRX; (2) the LHDI amplitude does not correlate well in time or space with the reconnection electric field, which is directly related to the rate of reconnection; and (3) significant LHDI amplitude persists in highcollisionality current sheets where the reconnection rate is classical. These findings suggest that the measured LHDI fluctuations do not play an essential role in determining the reconnection rate in MRX.
\end{abstract}

PACS numbers: 52.35.Vd, 52.35.Qz, 52.35.Ra, 52.72.+v

\footnotetext{
*Present address: Department of Physics and Astronomy, University of California, Los Angeles, CA 900951547; Electronic address: tcarter@physics.ucla.edu ${ }^{\dagger}$ Present address: Cymer, Inc., San Diego, CA
} 


\section{INTRODUCTION}

Magnetic reconnection [1] is a fundamental process in plasma which is thought to play important roles in both laboratory and natural plasmas through affecting magnetic topology and through heating and particle acceleration. It is generally accepted that the process of reconnection depends on the formation of sharp gradients in the magnetic field, called current sheets [2], which exist on scales where dissipation becomes important. The earliest quantitative model of current sheet reconnection was presented by Sweet and Parker [3], using the resistive magnetohydrodynamic (MHD) approximation. This model provides a mechanism by which magnetic topology can change much faster than would be allowed by simple resistive diffusion, but fails to explain the rapid timescales observed in natural and laboratory plasmas. The shortcoming of the Sweet-Parker model is the crucial dependence of the reconnection rate on the plasma resistivity. In collisionless plasmas where the Spitzer resistivity is small, very thin current sheets are needed in order for resistive dissipation to become important. The thinness of the current sheet impedes the flow of mass through the current sheet, slowing the reconnection process significantly.

One of the earliest proposals for speeding up Sweet-Parker reconnection was the inclusion of a turbulent anomalous resistivity in the resistive MHD model [4]. Strong currents and density gradients found in current sheets can drive microinstabilities which could provide sufficient anomalous dissipation to increase the Sweet-Parker rate to match observations. In addition to increasing the Sweet-Parker rate, simulations have found that using a currentdensity dependent turbulent resistivity may allow reconnection at nearly Alfvénic rates via the development of slow-mode shocks [5], as first proposed by Petschek [6]. While models using anomalous resistivity succeed in achieving fast reconnection, a crucial question to answer is: What microinstabilities operate in reconnecting current sheets, and can they provide sufficient resistivity to justify these models? A number of instabilities have been proposed to produce turbulence and anomalous resistivity in current sheets, including Buneman [7], electron cyclotron drift [8], ion acoustic [9], and lower-hybrid drift [10]. Due to the plasma parameters expected or observed in current sheets $\left(T_{\mathrm{i}} / T_{\mathrm{e}} \gtrsim 1, \beta \gtrsim 1, j / n e \sim v_{\mathrm{th}, \mathrm{i}}\right)$, many of these instabilities have been effectively ruled out as candidates for resistivity generation. Of the remaining candidates, the lower-hybrid drift instability (LHDI) is considered by some to be the "best bet" for providing anomalous resistivity in reconnecting current sheets [11]. 
However, it is well known that the LHDI is linearly stabilized by large plasma beta [12], suggesting that the instability might have difficulty being excited near the center of highbeta current sheets where resistivity generation is desired. Simulations have shown that the LHDI can grow in the edge of the current sheet, but disagree on the effectiveness of the instability in penetrating high beta regions and providing resistivity [13, 14].

Recently, an alternative theoretical picture of fast reconnection has emerged from simulations employing two-fluid effects, particularly effects embodied in the Hall terms in the generalized Ohm's law [15]. In these simulations, the relaxation of reconnected field lines is seen to be governed by the whistler wave, rather than by the Alfvén wave as in MHD reconnection. Due to the dispersive nature of the whistler wave, this results in a lowaspect-ratio current sheet geometry which does not impede mass flow and hence allows fast reconnection [16]. The reconnection rate in these simulations is found to be independent of the type of dissipation available in the current sheet, making the model very attractive for reconnection in collisionless plasmas. Simulations supporting this theoretical picture have been primarily two-dimensional, artificially suppressing instabilities and perhaps suppressing anomalous resistivity which could potentially dominate over other effects in setting the reconnection rate. However, initial three-dimensional Hall MHD simulations indicate that while turbulence does develop, the laminar 2D picture of reconnection remains intact and the turbulence only serves to slow the reconnection process [14].

The controversy over the role of turbulence in reconnection is obvious in the differences between these theoretical models. Motivated by the goal of resolving this controversy, we present in this paper experimental studies of fluctuations in the current sheet of the Magnetic Reconnection Experiment (MRX) [17]. The goal of this work was to: (1) identify and characterize any microinstabilities present in the MRX current sheet, and (2) determine the role of these instabilities during reconnection. Measurements of fluctuations in MRX were done primarily using floating Langmuir probes, but initial studies with magnetic pick-up probes were also performed. The result of these studies was the observation of broadband electrostatic and magnetic fluctuations in the current sheet which are identified as lowerhybrid drift waves $[18,19]$. This identification is made following careful examination of the frequency spectrum, spatial amplitude profile, and spatial correlation characteristics of the turbulence. The fluctuations are observed to develop with the formation of the current sheet in MRX and have a frequency spectrum centered near the local value of the lower 
hybrid frequency. The radial profile of the fluctuation amplitude is found to be peaked on the inner edge of the MRX current sheet, consistent with the computed profile of the LHDI linear growth rate. Two-point correlation measurements reveal a decorrelation length in the fluctuations comparable with the predicted wavelength of the LHDI, consistent with the expected strong linear growth rate in MRX current sheets $\left(\gamma \sim \omega_{\mathrm{r}}\right)$. The identification of the LHDI has provided an opportunity to experimentally investigate the role of this instability in reconnection. The radial amplitude profile, time behavior, and collisionality dependence of the fluctuations suggest that the LHDI is not contributing significantly to determining the reconnection rate in $\mathrm{MRX}$.

This paper is organized as follows: Section II offers a review of theories of the lowerhybrid drift instability, including the development of a model to be used to compare with measurements in MRX. In Section III the experimental apparatus and diagnostics used in this work are described. Section IV presents a detailed analysis of the fluctuation measurements and a discussion of their implications for reconnection in MRX. A summary of the work is offered in Section V.

\section{REVIEW OF THE LOWER-HYBRID DRIFT INSTABILITY}

The lower-hybrid drift instability has been studied theoretically for decades, motivated by its possible role in magnetic reconnection [10], theta-pinches and other fusion devices [20], and space plasmas (e.g. the magnetosphere [21]). In this section, a review of the theory and prior experimental studies of this instability is presented. Section II A reviews the theory of the LHDI, including the derivation and discussion of a local, linear, electrostatic model of the LHDI. This model will be utilized to explain features of the experimental data in Section IV. Section IIE briefly discusses prior observational studies of the LHDI.

\section{A. Linear LHDI Theory}

In order to develop a model to be used to compare with data taken on the MRX experiment, we present a derivation of a local, linear, electrostatic theory of the lower-hybrid drift instability. The following closely follows the procedures used by Krall and Rosenbluth [22], Davidson et al. [12], and Huba and $\mathrm{Wu}$ [23] in deriving the dispersion relation for the 
lower-hybrid drift instability. We consider a local model of the current sheet, assuming that the wavelength of the mode of interest is much smaller than the gradient scale length in the plasma, $\lambda \ll(d \ln n / d x)^{-1},(d \ln B / d x)^{-1}$. We therefore use a slab model in the derivation, with density and magnetic field gradients in the $x$ direction (corresponding to $r$ in MRX) and current in the $y$ direction (corresponding to $\theta$ in MRX). As the MRX current sheet thickness is comparable to $\rho_{\mathrm{i}}$ [24], we treat the ions as unmagnetized with a cross-field flow velocity $V$. The derivation will take place in the frame where the electric field is zero, and the ion flow velocity, $V$, therefore represents both the ion diamagnetic drift speed and any $E \times B$ electron current. The equilibrium ion distribution function is chosen to be a shifted Maxwellian:

$$
f_{\mathrm{i}}^{0}=\frac{n}{\pi^{3 / 2} v_{\mathrm{th}, \mathrm{i}}^{3}} \exp \left(-\frac{v_{\mathrm{x}}^{2}+\left(v_{\mathrm{y}}-V\right)^{2}+v_{\mathrm{z}}^{2}}{v_{\mathrm{th}, \mathrm{i}}^{2}}\right)
$$

Where $v_{\mathrm{th}, \mathrm{i}}=\sqrt{2 T_{\mathrm{i}} / M}$ and $n$ are evaluated locally. The electrons are magnetized, and we write the equilibrium distribution function as a function of the constants of the electron motion: $v^{2}, p_{z}$, and $p_{y}=m v_{y}-e A_{y}(x) / c$. If we assume that the gradient in the magnetic field is weak, we can approximate $p_{y} \approx m v_{y}-e B_{\mathrm{o}} x / c$. The electron distribution function is chosen to be:

$$
f_{\mathrm{e}}^{0}=\frac{n(X)}{\pi^{3 / 2} v_{\mathrm{th}, \mathrm{e}}^{3}} \exp \left(-\frac{v_{\perp}^{2}+v_{\mathrm{z}}^{2}}{v_{\mathrm{th}, \mathrm{e}}^{2}}\right)
$$

Where $X$ is related to the canonical momentum in the $y$ direction, $X=x-v_{y} / \Omega_{\mathrm{e}}=$ $-(e B / c) p_{y}$. We choose a local model and expand about $x=0$ to find:

$$
f_{\mathrm{e}}^{0} \approx\left(1-\epsilon_{\mathrm{n}} \frac{v_{y}}{\Omega_{\mathrm{e}}}\right) F_{\mathrm{m}, \mathrm{e}}
$$

Where $\epsilon_{\mathrm{n}}=d \ln n / d x$ and $F_{\mathrm{m}, \mathrm{e}}$ is a Maxwellian electron distribution.

Using the electrostatic approximation, the perturbed electron density can be shown to be (see Appendix A):

$$
\delta n_{\mathrm{e}}=-\frac{2 q n_{\mathrm{o}}}{m v_{\mathrm{th}, \mathrm{e}}^{2}} \tilde{\phi}-\frac{2 q n_{\mathrm{o}}}{m v_{\mathrm{th}, \mathrm{e}}^{2}} \tilde{\phi}\left(\omega-k_{y} v_{\mathrm{D}, \mathrm{e}}\right) \frac{2}{k_{\|} v_{\mathrm{th}, \mathrm{e}}} \int x d x \exp \left(-x^{2}\right) J_{0}^{2}\left(k_{\perp} \rho_{\mathrm{e}} x\right) \mathrm{Z}\left(\frac{\omega-k_{y} \bar{V}_{\nabla \mathrm{B}} x^{2}}{k_{\|} v_{\mathrm{th}, \mathrm{e}}}\right)
$$

The perturbed ion density is straightforward to calculate in the limit of unmagnetized, drifting ions, yielding [25]:

$$
\delta n_{\mathrm{i}}=\frac{2 q n_{\mathrm{o}}}{M v_{\mathrm{th}, \mathrm{i}}} \tilde{\phi}\left[1+\zeta_{\mathrm{i}} \mathrm{Z}\left(\zeta_{\mathrm{i}}\right)\right]
$$


Where $\zeta_{\mathrm{i}}=\left(\omega-k_{\mathrm{y}} V\right) / k v_{\text {th,i }}$. We can then use Poisson's equation to relate the density perturbations to the potential perturbation:

$$
\begin{aligned}
-k^{2} \phi= & 4 \pi e\left(\delta n_{\mathrm{i}}-\delta n_{\mathrm{e}}\right) \\
= & \phi\left\{-\frac{1}{2 \lambda_{\mathrm{d}, \mathrm{i}}^{2}} \mathrm{Z}^{\prime}\left(\zeta_{\mathrm{i}}\right)+\frac{1}{\lambda_{\mathrm{d}, \mathrm{e}}^{2}}\left(1+\frac{2\left(\omega-k_{y} v_{\mathrm{D}, \mathrm{e}}\right)}{k_{\|} v_{\mathrm{th}, \mathrm{e}}} \times\right.\right. \\
& \left.\left.\int x d x \exp \left(-x^{2}\right) J_{0}^{2}\left(k_{\perp} \rho_{\mathrm{e}} x\right) \mathrm{Z}\left(\frac{\omega-k_{y} \bar{V}_{\nabla \mathrm{B}} x^{2}}{k_{\|} v_{\mathrm{th}, \mathrm{e}}}\right)\right)\right\}
\end{aligned}
$$

The dispersion relation can then be obtained from roots of the following expression:

$$
\begin{aligned}
0= & 1-\frac{1}{2 k^{2} \lambda_{\mathrm{d}, \mathrm{i}}^{2}} \mathrm{Z}^{\prime}\left(\zeta_{\mathrm{i}}\right)+\frac{1}{k^{2} \lambda_{\mathrm{d}, \mathrm{e}}^{2}}(1+\psi) \\
\psi= & \frac{2\left(\omega-k_{y} v_{\mathrm{D}, \mathrm{e}}\right)}{k_{\|} v_{\mathrm{th}, \mathrm{e}}} \times \\
& \int x d x \exp \left(-x^{2}\right) J_{0}^{2}\left(k_{\perp} \rho_{\mathrm{e}} x\right) \mathrm{Z}\left(\frac{\omega-k_{y} \bar{V}_{\nabla \mathrm{B}} x^{2}}{k_{\|} v_{\mathrm{th}, \mathrm{e}}}\right)
\end{aligned}
$$

The value of the electron $\nabla B$ drift velocity can be shown to depend on the value of the total plasma beta. If we assume equilibrium between the magnetic and plasma pressure, and also that the temperature is spatially uniform, we find:

$$
\begin{aligned}
\nabla\left(\frac{B^{2}}{8 \pi}\right) & =-\nabla p \\
\frac{2 B^{2}}{8 \pi} \frac{\partial \ln B}{\partial x} & =-n\left(T_{\mathrm{e}}+T_{\mathrm{i}}\right) \frac{\partial \ln n}{\partial x} \\
\epsilon_{\mathrm{b}} & =-\frac{1}{2} \beta \epsilon_{\mathrm{n}} \\
\therefore \bar{V}_{\nabla B} & =-\frac{\beta}{2} v_{\mathrm{D}, \mathrm{e}}
\end{aligned}
$$

Where $\beta$ is the total plasma beta, $\beta=8 \pi n\left(T_{\mathrm{e}}+T_{\mathrm{i}}\right) / B^{2}$. Thus the plasma beta enters into equation 2 through the $\nabla B$ drift term in the plasma dispersion function.

Using equation 2, we can explore the linear characteristics of the LHDI using parameters relevant to the MRX experiment. The relevant dimensionless parameters in MRX are $\epsilon_{\mathrm{n}} \rho_{\mathrm{i}} / 2 \sim 1$ (density gradient scale length is roughly $\left.2 \rho_{\mathrm{i}}\right), V / v_{\mathrm{th}, \mathrm{i}} \sim 2.5\left(\mathrm{j} / \mathrm{ne}-n e v_{\mathrm{D}, \mathrm{e}} \sim\right.$ $\left.2.5 v_{\mathrm{th}, \mathrm{i}}\right)$, and $T_{\mathrm{i}} / T_{\mathrm{e}} \sim 1$. Figure 1 shows the real frequency and growth rate as a function of normalized wavenumber, $k_{\perp} \rho_{\mathrm{e}}$, for these parameters and for a few values of the normalized 
parallel wavenumber, $k_{\|} / k_{\perp} \sqrt{M / m_{\mathrm{e}}}$, for the case $\beta=0$. The frequencies obtained from roots of equation 2 are Doppler shifted by $k_{\perp} V$ in this plot in order to show the frequency in the ion rest frame. In the ion rest frame, positive real frequency is found for $k_{\perp}$ in the electron diamagnetic direction, indicating that the unstable waves propagate in that direction. The growth rate of the LHDI is found to be quite strong, and peaked near $k_{\perp} \rho_{\mathrm{e}} \sim 1$ and $\omega \sim \omega_{\mathrm{LH}}$. Significant growth is found at a wide range of $k_{\perp} \rho_{\mathrm{e}}$, translating to a range of real frequencies up to two to three times the lower hybrid frequency. In the ion rest frame, the phase velocity of the waves at peak growth $\left(\omega \sim \omega_{\mathrm{LH}}, k_{\perp} \rho_{\mathrm{e}} \sim 1\right)$ is:

$$
\frac{\omega}{k_{\perp}} \sim \omega_{\mathrm{LH}} \rho_{\mathrm{e}}=\sqrt{\frac{T_{\mathrm{e}}}{T_{\mathrm{i}}}} v_{\mathrm{th}, \mathrm{i}}
$$

Therefore the strongest growth is found where ion Landau damping of the waves is strongest. This actually drives growth of the LHDI, as it is a negative energy drift wave in the ion rest frame [26]. For $k_{\|}=0$, the growth rate of the waves is due to $-\left(\partial f_{\mathrm{i}}^{0} / \partial v\right)_{\omega / k}$ and no damping is provided by the electrons (for $\beta=0$ ). As $k_{\|}$is acquired by the wave, access to electron Landau damping along the field line is provided, resulting in a lowering and eventual suppression of the growth rate, at very small values of $k_{\|} / k_{\perp}$.

Figure 1 is for the case of zero plasma beta. Significant beta values are found in the MRX current sheet, from roughly 10\%-100\% at the edge to infinite local beta at the field null in guide-field-free current sheets. Figure 2 shows the effect of increasing plasma beta on the peak growth rate for the LHDI under the same conditions in Figure 1 and for $k_{\|}=0$. As beta is initially increased there is little change in the peak growth rate, but after $\beta \approx 1$, the peak growth rate drops dramatically.

The local, electrostatic, linear model of the LHDI presented in this section shows that we should expect the LHDI to be fairly strongly growing in conditions similar to those found in MRX, with $\gamma \sim \omega_{\mathrm{LH}}$ at $k_{\perp} \rho_{\mathrm{e}} \sim 1$. It is interesting to note that the marginal state of the LHDI is predicted to occur at significantly shallower density gradients than those observed in MRX. An estimate of the critical density gradient (assuming the crossfield current to be entirely diamagnetic) is $\rho_{\mathrm{i}} \epsilon_{\mathrm{n}} / 2 \sim 2 \Omega_{\mathrm{i}} \omega_{\mathrm{LH}} \sim 1 / 20$ [12]. The predicted LHDI growth rate drops dramatically as beta is raised, and the instability is likely to be suppressed in the center of the current sheet, where beta is locally infinite. Electromagnetic corrections to the LHDI were first explored by Davidson [12], who found these corrections to be destabilizing in regimes similar to those found in $\operatorname{MRX}\left(V / v_{\mathrm{th}, \mathrm{i}} \gtrsim 1\right)$. These destabilizing 
effects were found to lead to the restoration of growth at longer wavelengths, but only increased the value of the peak growth rate slightly compared to the electrostatic case. Thus, the overall effect of beta is a stabilizing one, however the electrostatic model presented in this section slightly overestimates the degree of stabilization. For this reason, as well as for simplicity, the electrostatic model presented above, which captures the dominate finitebeta effect of resonant $\nabla B$ stabilization, is used instead of a fully electromagnetic theory to calculate peak growth rates for comparison with data presented in Section IV. For $k_{\|}=0$, the electromagnetic LHDI is flute-like, only generating perturbations in the background magnetic field component ( $B_{\mathrm{z}}$ in MRX).

\section{B. Nonlinear effects and simulations}

\section{Saturation mechanisms}

The anomalous transport properties of the LHDI have been of great interest in the theoretical literature, especially as applied to theta pinches [27] and magnetic reconnection [28]. The starting point for estimates of transport coefficients is the determination of the saturation level of the LHDI. The earliest estimate of this level was done by Davidson [29], where quasilinear theory was employed to determine the efficiency of saturation by plateau formation and current relaxation. The former is unlikely to be important in MRX plasmas, as collisions are likely to maintain Maxwellian particle distribution functions (this has been observed spectroscopically [30]). It has been pointed out [26] that current relaxation does not provide a realistic bounds on the saturated amplitude, as the energy in the field is tied to the current, and this thermodynamic estimate should be based on the total magnetic energy in the system. Ion trapping was observed as the saturation mechanism for the LHDI in simulations by Winske [31]. This mechanism is effective when the LHDI spectrum is nearly monochromatic, as was observed in these simulations at moderate drift velocity $V / v_{\mathrm{th}, \mathrm{i}} \gtrsim 3$. Huba [32] considered the effect of electron resonance broadening on the satu-

ration of the LHDI. In this study, the stabilizing electron $\nabla B$ resonance was shown to be nonlinearly broadened, allowing a larger population of electrons to interact with and damp the LHDI waves. A saturation estimate for this process (however for $V / v_{\text {th }, \mathrm{i}} \lesssim 1$ ) was made 
by Gary [33]:

$$
\left(\frac{\mathcal{E}}{n T_{\mathrm{i}}}\right) \approx \frac{2}{5} \frac{m_{\mathrm{e}}}{M} \frac{\Omega_{\mathrm{e}}^{2}}{\omega_{\mathrm{p}, \mathrm{e}}^{2}}\left(\frac{T_{\mathrm{i}}}{T_{\mathrm{e}}}\right)^{1 / 4} \frac{V^{2}}{v_{\mathrm{th}, \mathrm{i}}^{2}}
$$

However, this saturation mechanism, which is similar to electron trapping, might be hampered in MRX by high electron collisionality. Finally, a numerical calculation of the effect of nonlinear Landau damping (or mode-mode coupling) on the saturation of the LHDI was performed by Drake [26]. In this case, nonlinear transfer of energy from growing long wavelength modes $\left(k \rho_{\mathrm{e}} \sim 1\right)$ to damped short wavelength modes provided a saturation mechanism. This calculation yielded an estimate for the saturation level of the LHDI:

$$
\frac{e \phi}{T_{\mathrm{i}}} \approx 2.4\left(\frac{2 m_{\mathrm{e}}}{M}\right)^{1 / 2} \frac{V}{v_{\mathrm{th}, \mathrm{i}}}
$$

This calculation ignored any nonlinear coupling into damped modes with finite $k_{\|}$, and therefore is likely to be an overestimate of the saturation amplitude.

\section{Quasilinear resistivity}

Davidson [27] presented a calculation of the anomalous resistivity and heating rates of the LHDI which will be reviewed here. The quasilinear equation for the evolution of the zero order distribution function of species $j$ due to the presence of waves in the plasma is:

$$
\left(\frac{\partial}{\partial t}+\mathbf{v} \cdot \frac{\partial}{\partial \mathbf{x}}+\frac{q_{j}}{m_{j}}\left(\frac{\mathbf{v} \times \mathbf{B}}{c}\right) \cdot \frac{\partial}{\partial \mathbf{v}}\right) f_{j}=\left(\frac{\partial f_{j}}{\partial t}\right)_{\text {anom }}=-\frac{q_{j}}{m_{j}}\left\langle\delta \mathbf{E} \cdot \frac{\partial \delta f_{j}}{\partial \mathbf{v}}\right\rangle
$$

The anomalous momentum exchange rate between species $j$ and the fluctuations can be calculated by taking the first velocity moment of $\left(\partial f_{j} / \partial t\right)_{\text {anom }}$ for velocity in the current direction:

$$
\left(\frac{\partial}{\partial t} n_{j} m_{j} V_{y, j}\right)_{\text {anom }}=q_{j}\left\langle\delta E_{y} \delta n_{j}\right\rangle
$$

Eqn. 6 can then be used to compute an effective collision rate due to the waves:

$$
\nu_{\mathrm{eff}}=\frac{q_{j}}{n_{j} m_{j} V_{y, j}}\left\langle\delta E_{y} \delta n_{j}\right\rangle
$$

Eqn. 7 provides an instability-model-independent way to experimentally determine the effective collision rate due to a measured spectrum of electric field and density fluctuations. However, simultaneous measurement of the amplitude and phase of both density and electric field fluctuations in a plasma is quite a difficult task, and was not attempted as part of this 
work. A simpler, yet model-dependent, expression for the effective collision rate can be obtained through using the linear theory for the LHDI to compute the density perturbation as a function of the electric field perturbation, $\delta n_{j}=-\chi_{j} i k_{y} \delta E_{y, k_{y}} / 4 \pi q_{j}$. Using the expression for the ion density perturbation in Eqn. 1, the effective collision rate estimate reduces to $[27]:$

$$
\nu_{\mathrm{LHDI}}=\operatorname{Im}\left[k_{\perp} \frac{4 \omega_{\mathrm{p}, \mathrm{i}}^{2}}{k_{\perp}^{2} v_{\mathrm{th}, \mathrm{i}}^{2}} \zeta_{\mathrm{i}} \mathrm{Z}\left(\zeta_{\mathrm{i}}\right)\right]_{k_{\perp, \max }} \frac{T_{\mathrm{i}}}{m_{\mathrm{e}} V} \frac{\mathcal{E}}{n T_{\mathrm{i}}}
$$

Where $k_{\perp, \text { max }}$ indicates that the expression should be evaluated at the frequency and wavenumber at peak growth, and $\mathcal{E}=(\delta E)^{2} / 8 \pi$. Experimental evaluation of Eqn. 8 can be performed with knowledge of only the amplitude of the electric field fluctuations in the plasma.

\section{Review of simulations of the LHDI}

Although predictions of strong anomalous resistivity due to the LHDI have been made, the usefulness of this resistivity in reconnection is questionable if the LHDI is suppressed at the center of high-beta current sheets, where it would be needed to provide dissipation. Several simulations have been performed to study the LHDI and investigate the likelihood of the it penetrating to the center of a current sheet[see, e.g. 13, 14, 34-37]. Two recent simulation efforts addressing the LHDI during magnetic reconnection disagree on the importance of the instability in determining the reconnection rate. The first effort involves three-dimensional particle simulations of reconnection performed by Horiuchi and Sato [13]. In these simulations the LHDI grows up early on the edges of the current sheet, consistent with finite-beta stabilization. In the case that no external driving electric field was present, the LHDI did not penetrate further into the current sheet, but instead resulted in a modification of the current sheet profile which in turn drove a low-frequency magnetic instability. In this case, reconnection electric field was not induced at the null by the LHDI but was instead provided by the low-frequency instability, which was seen to generate significant anomalous resistivity. The low-frequency instability was identified as the drift-kink instability (DKI), which was so named by Zhu and Winglee [38] after observations in simulations of the magnetotail, but which was perhaps first studied analytically by Yamanaka [39] (and later by Winske [40]). When a driving electric field was applied in the simulations by Horiuchi and Sato, 
the LHDI was found to penetrate to the magnetic null and provide anomalous dissipation prior to the triggering of the DKI. In either case, the LHDI was seen as quite essential to determining the reconnection rate in these simulations, either through penetration to the null line or through nonlinearly driving the DKI. It should be noted that the importance of the DKI in current sheets is currently the topic of much theoretical debate. The DKI has been primarily observed in low mass ratio particle simulations, and Daughton [41] has shown that while the growth rate of this instability can be large when the mass ratio is artificially small, the DKI should have negligible growth rate at realistic mass ratios in Harris equilibria. Daughton does however suggest that other equilibrium profiles, especially those with significant background density, may increase the growth rate of the DKI [41, 42] .

A second recent simulation effort has shown that effects associated with the Hall term in the generalized Ohm's law can result in fast reconnection in laminar current sheets[15]. The simulations supporting this fast reconnection mechanism have been almost exclusively done in two-dimensions (the $x-z$ plane in the model presented in Section II A), artificially suppressing instabilities like the LHDI. However, recent 3D simulations by Rogers et al. [14] using a Hall MHD model have shown that while LHDI does develop in the edge of the current sheet, it does not dramatically alter the physical picture of fast reconnection found in the 2D simulations. In fact, development of the LHDI was observed to slow the reconnection rate relative to the rate found in laminar 2D simulations. The differing conclusions reached by these two simulation efforts demonstrate the theoretical controversy over the role of turbulence, specifically due to the LHDI, in reconnection.

\section{E. Prior experimental studies of the LHDI}

There have been very few experimental observations of the LHDI, and none in previous laboratory reconnection experiments. The earliest report of an experimental observation of the LHDI was made by Gurnett et al. [43], who studied satellite measurements of fluctuations

in the Earth's magnetotail. Analysis by Huba et al. [28] suggested that the frequency spectrum and amplitude of the waves was consistent with the operation of the LHDI in the magnetotail. Shinohara et al. [11] also presented an analysis of recent satellite measurements in the magnetotail, suggesting that the observed fluctuations were due to the LHDI. An estimate of the anomalous resistivity due to these fluctuations was made, but it was found 
that the value of this resistivity was not enough to increase the growth rate of the tearing mode to the level necessary to explain the triggering of an associated substorm. However, Shinohara et al. suggested that the computed anomalous resistivity might still be enough to be essential to magnetic reconnection in the tail. In these satellite measurements, detailed observation and analysis of the LHDI is quite difficult, as the profile and location of the tail current sheet is not well measured simultaneously to the fluctuation measurements. In both cases, however, the data suggested that electrostatic fluctuations might be strongest away from the center of the current sheet. There have been experimental studies of the LHDI in other plasma configurations which are not directly relevant to the problem of magnetic reconnection. $\mathrm{A} \mathrm{CO}_{2}$ laser scattering measurement of fluctuations in a thetapinch plasma was made by Fahrbach et al. [44]. These measurements provided some limited information on the wavelength and frequency spectrum of fluctuations in the plasma, and the characteristics were shown to be consistent with linear and nonlinear theories of the LHDI [26]. Measurements in magnetoplasmadynamic (MPD) thrusters, which involve strong crossfield current and density gradients, have also revealed evidence for the LHDI [45, 46].

\section{EXPERIMENTAL APPARATUS}

The measurements reported in this paper were taken on the Magnetic Reconnection Experiment (MRX) [17] at Princeton Plasma Physics Laboratory. MRX was constructed for the purpose of studying magnetic reconnection in a well-controlled laboratory plasma where MHD is satisfied in the bulk of the plasma (Lundquist number $(S) \gg 1, \rho_{\mathrm{i}} \ll L$ ). A schematic drawing of the MRX apparatus, showing location of the current sheet and fluctuation measurement geometry, is shown in Figure 3.

Current sheets in MRX are formed between two coilsets called "flux cores", shown in Figure 3. The current sheet is indicated by x's in the figure, surrounded by a representative field line. The primary sheet current flows in the toroidal $(\theta)$ direction, the reconnecting field is in the $\mathrm{Z}$ direction, and the density and magnetic field gradients are in the $\mathrm{R}$ direction. The work reported in this paper was done in current sheets where no macroscopic $B_{\theta}$ (sometimes called "guide" field) is present during reconnection ("null-helicity" reconnection, as opposed to "co-helicity", where a guide field is present).

The free energy sources available to drive instabilities in MRX current sheets can be 
at least partially revealed through the measurement of profiles of magnetic field (and hence current) and plasma temperature and density. The knowledge of these profiles will also allow theoretical prediction of instability characteristics in MRX for comparison with fluctuation measurements. The bulk of the diagnostics in the MRX device are for the measurement of magnetic fields, with close to 180 magnetic pickup coils in the vacuum vessel. These coils are hand wound, using 80 turns of 38 gauge magnet wire on $3 \mathrm{~mm}$ diameter, $3 \mathrm{~mm}$ long cylindrical plastic forms. The coils are distributed among three probes; two of these probes (90-channel and 60-channel 2D probes) are for the purpose of measuring all three vector components of the field in a coarse grid spacing $(4 \mathrm{~cm}$ near the current sheet, and 6 or $8 \mathrm{~cm}$ at radii well inside the current sheet location) in one toroidal plane of the experiment. Using these magnetic measurements and assuming axisymmetry, the poloidal flux and electric field can be calculated: $\psi=\int_{0}^{R} 2 \pi r B_{\mathrm{z}}(r) \mathrm{d} r, E_{\theta}=-1 /(2 \pi R)(\partial \psi / \partial t)$. A high-resolution $(0.5 \mathrm{~cm}$ spacing) 1D magnetic probe is used to measure $B_{\mathrm{z}}$ along the $r$ direction. The magnetic field profile in MRX is well described by the Harris sheet theoretical equilibrium profile [24, 47]. The measured $B_{\mathrm{z}}$ field in MRX is fit to this theoretical profile $\left(B \propto \tanh \left(\left(r-r_{\mathrm{o}}\right) / \delta\right)\right)$ and the current density is derived analytically from the fit. The thickness of the current sheet in MRX is found to be comparable to both the ion skin depth and the ion gyroradius $\left(\delta \sim \rho_{\mathrm{i}} \sim c / \omega_{\mathrm{p}, \mathrm{i}}\right)[24]$. A triple Langmuir probe [48] is employed to measure density $\left(n_{\mathrm{e}}\right)$, electron temperature $\left(T_{\mathrm{e}}\right)$, and floating potential $\left(V_{\mathrm{f}}\right)$ profiles in the current sheet.

Collisionality in MRX current sheets is characterized by the parameter $\lambda_{\mathrm{mfp}} / \delta$, where $\delta$ is the width of the current sheet and $\lambda_{\operatorname{mfp}}$ is the electron mean free path against Coulomb collisions. Two observations which motivate the study of turbulence and anomalous resistivity have been made as the collisionality was lowered $\left(\lambda_{\mathrm{mfp}} / \delta\right.$ is increased) in MRX. The first observation is that the measured toroidal reconnection electric field, $E_{\theta}$, is no longer balanced by classical collisional drag at the center of the current sheet, $E_{\theta} / \eta_{\mathrm{sp}} j_{\theta} \gg 1$, where $\eta_{\mathrm{sp}}$ is the classical Spitzer perpendicular resistivity [49]. If the measured ratio $E / j$ is defined as an effective resistivity, $\eta^{*}=E / j$, reconnection data from MRX is found to agree with a generalized Sweet-Parker theory based on this effective resistivity (and also including compressibility and downstream pressure)[50]. The second observation is that of direct, nonclassical ion heating during reconnection in MRX current sheets [51]. One possible explanation for these two observations is the presence of turbulence in low-collisionality MRX current sheets, which creates a turbulent anomalous resistivity $\eta^{*}>\eta_{\mathrm{sp}}$ (so that $E_{\theta} / \eta^{*} j_{\theta}=1$ ) 
and directly heats the ions.

The initial search for high-frequency fluctuations in MRX using probes revealed broadband noise generated by impedance mismatches in both power transmission lines from the MRX capacitor banks and in transmission lines of the diagnostics themselves. This noise precluded the detection of signals from the plasma and had to be addressed in order to study fluctuations in the MRX current sheet. In order to improve immunity to noise generated by the power circuitry in MRX and to facilitate active impedance matching in the diagnostics, small broadband buffer amplifiers were built into probes used for the fluctuation studies reported here. The use of a miniature SOT-23 surface mount package for the buffer (BurrBrown OPA682), along with 0805 package surface mount capacitors and resistors, allowed the placement of all components on a double-sided printed circuit board of $4.5 \times 50 \mathrm{~mm}$ approximate dimensions. The boards are placed inside $1 / 4 "(0.635 \mathrm{~cm})$ to $3 / 8 "(0.9525 \mathrm{~cm})$ stainless probe shafts, which allows the leads connecting the probe tips to the amplifier to be only several millimeters $(5-10 \mathrm{~mm})$ long. The amplifier allows an easy transition from the high-impedance probe tip into a $50 \Omega$ transmission line, eliminating impedance matching issues. While the overall voltage gain is unity, the amplifier does boost the signal current to assist in noise immunity. A high-bandwidth ferrite core 1:1 pulse transformer is used to provide isolation from the plasma in electrostatic (Langmuir probe) diagnostics, but is not present in amplified magnetic pick-up coil diagnostics. The magnetic field value of the core saturation is well above the fields used in these experiments ( 200G). Perturbation of the background field due to the presence of a high- $\mu$ ferritic material is negligible due to the size and toroidal geometry of the transformer. Signals are propagated down the probe shafts using low-loss semi-rigid coaxial line (UT-85LL). Signal transport from the probe to the digitizing oscilloscope (approximately 12m away) is accomplished using low-loss RG8 coaxial cable. The bandwidth of the system (amplifier input to RG8 output) is measured to be $100 \mathrm{kHz} \lesssim f \lesssim 125 \mathrm{MHz}$ when the transformer is used for isolation and $f \lesssim 300 \mathrm{MHz}$ when no transformer is used.

Fluctuations in the plasma floating potential were measured using differential floating Langmuir probes. Floating potential measurements were chosen over ion saturation current measurements due to the difficulty of measuring high frequency current signals accurately in the presence of cable capacitance. Differential measurement was performed in order to remove low frequency floating potential signals, which can be on the order of $100 \mathrm{~V}$ 
(fluctuating signals are on the order of $1 \mathrm{~V}$ ). Single floating probe measurements using voltage division were not practical due to the limited dynamic range of the data acquisition system (8 bit) and noise generated by the high-power pulsed electronics. Differential floating Langmuir probes are constructed using two spatially separated cylindrical tungsten wires sheathed in alumina $\left(\mathrm{Al}_{2} \mathrm{O}_{3}\right)$. The diameter of the tungsten tips varied from $30 \mathrm{mil}(0.762 \mathrm{~mm})$ to $5 \mathrm{mil}$ $(0.127 \mathrm{~mm})$, and in all probe tips a $1 \mathrm{~mm}$ length of the wire is exposed to the plasma. Studies of spatial correlations were performed using three-tip probes, where two of the tips are used to make spatially-separated differential floating potential measurements using the third tip as a common reference. Correlation probes were constructed with 1, 3.5, and $10 \mathrm{~mm}$ separation. Magnetic field fluctuations are measured using magnetic pick-up coil based probes. The coils are placed inside small glass tubes coated with graphite to provide electrostatic shielding. The pick-up probes are coupled directly to a buffer amplifier at the end of the probe shaft, with no transformer. The bandwidth of the probe is set by the $L / R$ time of the coil $(L \approx 10 \mu \mathrm{H})$ based on the input impedance of the buffer amplifier $(500 \Omega)$, which is around 20ns (making the bandwidth $f \lesssim 50 \mathrm{MHz}$ ). The probes were inserted radially into MRX plasmas, as shown in Figure 3.

\section{MEASUREMENTS OF FLUCTUATIONS IN THE MRX CURRENT SHEET}

Measurements of fluctuations in the current sheet of MRX are reported in this section. While fluctuations have been studied in current sheets previously [52], the measurements reported here are the first to be done in a current sheet formed in a plasma where, on the global scale, ions are magnetized $\left(\rho_{\mathrm{i}} \ll L\right)$ and the MHD approximation is satisfied $(S \gg 1)$. In addition, fast reconnection, enhanced resistivity, and non-classical ion heating have been observed in MRX [50, 51], providing an opportunity to determine if turbulence plays an essential role in these phenomena. Measurements of high-frequency fluctuations were performed in the current sheet of MRX, with the following goals: (1) Identify any instabilities present in the current sheet and determine their characteristics and (2) determine the influence of these instabilities on the process of reconnection in MRX. These measurements resulted in the first observation of the lower-hybrid drift instability in a laboratory current sheet. This instability has been studied theoretically for decades in the context of current sheets and magnetic reconnection, yet no detailed experimental investigation of the 
instability has been possible until this work.

\section{A. Observation of the lower-hybrid drift instability}

Measurements using amplified floating double Langmuir probes placed on the edge of current sheets in null-helicity discharges in MRX have revealed the presence of broadband fluctuations near the lower hybrid frequency. In this section, evidence supporting the identification of these fluctuations as lower-hybrid drift waves is presented. The evidence is provided by detailed studies of the frequency spectrum, radial amplitude profile, and spatial correlations and propagation characteristics of the fluctuations. These observations will be shown to be consistent with theoretical predictions for the lower-hybrid drift instability, using the theory developed in Section II for comparison.

Figure 4 shows an example of an differential floating potential signal $\left(\delta \phi_{\mathrm{f}}\right)$ taken at $r=0.34 \mathrm{~m}$ (refer to Figure 3 for measurement geometry), along with a time trace of the total toroidal plasma current during a hydrogen discharge in MRX. The plasma current rises during formation of the current sheet in MRX and then typically flattens in time during the quasi-steady period of magnetic reconnection. The fluctuations are seen to arise with the formation of the current sheet and persist for $10-20 \mu s$. The amplitude of the measured fluctuations is typically several hundred millivolts, but can be as high as $1-2 \mathrm{~V}$. A normalized fluctuation amplitude can be constructed by comparing the amplitude to the measured electron temperature, $e \delta \phi_{\mathrm{f}} / T_{\mathrm{e}}$. This normalized amplitude is typically found to be several percent $\left(T_{\mathrm{e}} \sim 5-10 \mathrm{eV}, e \delta \phi_{\mathrm{f}} / T_{\mathrm{e}} \lesssim 10 \%\right)$. A windowed FFT of the shown example

signal is inset in Figure 4, with a vertical line marking the position of the time averaged lower hybrid frequency, $f_{\mathrm{LH}} \sim 16 \mathrm{MHz}$. The FFT is performed using a Hanning window, $8 \mu s$ wide about $t=252 \mu \mathrm{s}$, and the plot is made using a linear vertical axis. The lower hybrid frequency is determined from measurements of the magnetic field near the fluctuation probe using $\omega_{\mathrm{LH}}=\sqrt{\Omega_{\mathrm{e}} \Omega_{\mathrm{i}}}$.

\section{Frequency spectrum}

The LHDI is expected to produce fluctuations whose frequency spectrum is located near the lower hybrid frequency. The detailed dependence of the frequency spectrum of the 
measured floating potential fluctuations on the lower hybrid frequency was explored through varying the peak field in the current sheet and the mass of the working gas $\left(f_{\mathrm{LH}} \propto B / \sqrt{M}\right)$. The peak magnetic field value was varied through raising or lowering the voltage on the capacitor bank used to generate the poloidal field. Using this technique, the peak field was scanned from roughly $100 \mathrm{G}$ (using $10 \mathrm{kV} / 8 \mathrm{kV}$ on the toroidal field/poloidal field bank) to $300 \mathrm{G}(14 / 12 \mathrm{kV})$. Both hydrogen and helium were used as working gases, allowing for a factor of 2 change in the lower hybrid frequency due to ion mass.

Figure 5(a) shows a set of example average floating potential fluctuation power spectra (linear vertical axis, logarithmic horizontal axis) at different local field values in hydrogen.

Each plot is generated through averaging the spectrum of 10 discharges whose local magnetic field value falls within a $50 \mathrm{G}$ window of the magnetic field value annotating the graph. There is an upward shift evident in the power spectrum with increasing field strength, consistent with the shift in the local lower hybrid frequency. Figure 5(b) shows the frequency of peak fluctuation amplitude versus local magnetic field for discharges in hydrogen. While there is some scatter, the peak frequency is seen to increase with increasing magnetic field in a manner consistent with the lower hybrid frequency. Figure 6 shows the average power spectrum of the fluctuations for hydrogen and helium discharges with similar local magnetic field. A clear downshift in the location of the fluctuation spectrum is observed in helium discharges, again consistent with the change in the local lower hybrid frequency.

The theory of the LHDI predicts that the peak of the growth rate should occur at a wavenumber associated with a real frequency of roughly the lower hybrid frequency. The observed frequency spectrum is consistent with the linear theory in this regard, as the peak is near the lower hybrid frequency. The LHDI theory also predicts a fairly wide range of wavenumbers where appreciable growth is found, as shown in Figure 1, which is consistent with the observation of a wide frequency range in the fluctuation spectra.

\section{Spatial amplitude profiles and time behavior}

The LHDI is expected to be driven by density gradients and cross-field currents, which would suggest that it might be localized near these energy sources in MRX current sheets. In order to determine if the observed fluctuations are consistent with these expectations, a study of the radial amplitude profile was performed. A comparison with the linear theory 
developed in Section II is presented, and provides further support for the conclusion that the measured fluctuations are due to the LHDI. A discussion of the observed time behavior of the fluctuations, also based on the linear theory, is provided.

Radial profiles of the amplitude of the floating potential fluctuations were constructed through shot-to-shot positioning of the probe and averaging over many shots at each position. Figure 7 shows average radial profiles of the root-mean-square fluctuating floating potential amplitude superimposed on the computed average current density profile at four times during a set of more than 200 low-collisionality $\left(\lambda_{\mathrm{mfp}} / \delta \sim 5-10\right)$ MRX discharges $(12 / 10 \mathrm{kV}, 4 \mathrm{mT}$ fill pressure, hydrogen). The current densities shown are computed by first fitting the average measured magnetic field profile to a Harris sheet profile, then deriving the current density from the fit. The magnetic measurements shown are made at a small toroidal separation $\left(10-15^{\circ}\right)$ from the fluctuation diagnostic. As the current sheet is formed, the width of the sheet thins to be comparable to the ion skin depth while the radial position of the current sheet moves outward (due to the hoop force) in order to establish equilibrium with an applied steady-state magnetic field in the $z$-direction. The plotted fluctuation amplitude is determined through first high-pass filtering individual fluctuation measurements (digitally), then averaging the square amplitude at each radial position. The error bars represent shotto-shot variations in the measurement. The fluctuations are observed to grow up on the inner edge of the current sheet, then strengthen and track the current sheet as it moves toward an equilibrium position. Later in time, the amplitude decays fairly rapidly even though the current sheet persists and reconnection continues.

Figure 8 shows a contour plot in the $r-t$ plane of the RMS floating potential amplitude, along with the trajectory of the center of the current sheet and sheet thickness $\left(R_{\mathrm{o}}\right.$ and $R_{\mathrm{o}} \pm \delta$, determined from the fit of the average magnetic field to a Harris profile). This figure shows in more detail how the fluctuation amplitude follows the trajectory of the current profile. As reconnection proceeds, the equilibrium is altered by the depletion of flux inside the current sheet. This lowers the $B^{2}$ pressure pushing out on the current sheet, resulting in an inward shift of the equilibrium position, as shown in the figure after $t \approx 258 \mu \mathrm{s}$. Reconnection continues as the current sheet moves inward, until roughly $t=280 \mu \mathrm{s}$.

The radial profile measurements raise a key question: Why is the radial amplitude profile asymmetric? We will address this question using the linear electrostatic model of the LHDI derived in Section II. Linear calculations of the local growth rate profile of the LHDI were 
performed based on measured profiles of density, electron temperature and magnetic field. Electron temperature and density profiles were acquired in a similar fashion to the fluctuation profile: through shot-to-shot positioning of a triple Langmuir probe and averaging over several shots (at least 10) per position. The triple Langmuir probe measured density profile at $t=264 \mu \mathrm{s}$, along with a Harris sheet fit to the measured average magnetic field profile, is shown in Figure 9(a). Both the magnetic field and the density are observed to be radially asymmetric with respect to the center of the current sheet. The magnetic field asymmetry is due to the cylindrical geometry of the field coils (flux cores) in MRX, which generate stronger fields inside the current sheet location than outside. The density asymmetry arises so that radial force balance can be achieved with this magnetic field profile [24]. The density gradient is a source of free energy for the LHDI, and a stronger gradient on the inner edge implies the growth rate should be larger there. In addition, the density asymmetry creates an radially asymmetric cross-field electron-ion flow speed difference, $V_{\mathrm{d}}=j / n e$. This cross-field drift is also an important drive for LHDI, and for a symmetric current density, the larger density on the outer edge produces a smaller flow difference between the ions and electrons. The combination of the density and magnetic field asymmetries produces a strong asymmetry in the profile of the electron beta $\left(\beta_{\mathrm{e}}=8 \pi n T_{\mathrm{e}} / B^{2}\right)$, as shown in Figure $9(\mathrm{~b})$. The beta on the inner edge of the current sheet is on the order of $10 \%$, compared to the outer edge which has near unity beta. The large beta on the outer edge should be has a significant stabilizing influence on the LHDI.

In order to compute a profile of maximum LHDI growth rate, a smooth fit to the density profile measurement (dotted line in Figure 9(a), arbitrarily using a Lorentzian with different "temperatures" on either side of the current sheet) along the fitted magnetic field and current profiles at $t=264 \mu \mathrm{s}$ were used to compute parameters in Equation 2 (assuming $\left.T_{\mathrm{i}} / T_{\mathrm{e}}=1,2,3\right)$. The cross-field ion velocity $(V)$ in this equation was determined by equating the plasma current density to $j=n e\left(V+v_{\mathrm{D}, \mathrm{e}}\right)$, where $v_{\mathrm{D}, \mathrm{e}}$ is the electron diamagnetic velocity. Dispersion relations and growth rates for the LHDI were then found through numerically finding roots of Equation 2, using model parameters determined from measured plasma parameters at each radial location. Figure $9(\mathrm{~d})$ shows the profile of the maximum growth rate (maximized over wavenumber) which resulted from these calculations. The predicted growth rate profile is quite asymmetric, in fact growing modes are only found on the inner edge of the current sheet. Growth is suppressed on the outer edge by the 
large beta, low ion drift speed, and small normalized density gradient. The growth rate profile compares well with the measured fluctuation amplitude profile at $t=264 \mu \mathrm{s}$, which is repeated in Figure 9(c) for clarity. There is no reason to expect quantitative agreement between the saturated amplitude of the fluctuations and the linear growth rate in this case. However, the linear growth rate profile should indicate where the drive for the instability is strongest and hence should suggest the saturated amplitude might be largest.

The amplitude of the fluctuations in this set of discharges is observed to decrease rapidly shortly after $t=265 \mu \mathrm{s}$. The radial profiles of measured plasma parameters change fairly smoothly by comparison, and therefore do not seem to provide an answer for the rapid timescale of the decrease. One unknown parameter in these experiments in hydrogen is the ion temperature. It is expected that the ions should be heated and the ion temperature should rise monotonically during reconnection, based on measurements in helium plasmas [30]. This ion heating could increase the $T_{\mathrm{i}} / T_{\mathrm{e}}$ ratio and also increase the total plasma beta. Davidson et al. [12] have shown that at normalized drift speeds $V_{\mathrm{d}} / v_{\mathrm{th}, \mathrm{i}} \gtrsim 1$ the critical beta at which the LHDI is suppressed can drop with increasing $T_{\mathrm{i}} / T_{\mathrm{e}}$. Figure $9(\mathrm{~d})$ shows some support for this in MRX parameter regimes as the calculated linear growth rate drops with increasing $T_{\mathrm{i}} / T_{\mathrm{e}}$. We expect that the ion temperature should be less than the electron temperature before reconnection begins, again based on previous measurements in helium. An estimate of the ion temperature at late times can be made through considering an MHD force balance across the current sheet, resulting in $T_{\mathrm{i}} / T_{\mathrm{e}} \gtrsim 2$ for $t=274 \mu \mathrm{s}$. The linear growth rate should drop somewhat due to an increase in the temperature ratio to $T_{\mathrm{i}} / T_{\mathrm{e}} \sim 2$ (see Figure 9), but this may not fully explain the observed greater than four-fold drop in the fluctuation amplitude.

We can offer some additional suggestions as to the source of the rapid decrease of the fluctuation amplitude observed in these discharges. A nonlocal theory is likely to be necessary to fully describe the LHDI in MRX current sheets, due to the presence of gradients in both the $r$ and $z$ direction. In particular, it is important to note that the local theory assumes that the strongest growing mode occurs at $k_{\|} \sim 0$, or at infinite parallel wavelength. The current sheet in MRX is, of course, of finite length and largest parallel wavelength is likely set by this length. It is possible that plasma conditions away from the center of the current sheet (along $z$ ) could have repercussions on the behavior of the instability near the center of the current sheet, due to the tendency for the instability to grow at large parallel 
wavelength. For instance, it is known that the plasma pressure downstream in the MRX current sheet builds up during reconnection [49], and this might lead to large downstream beta. This beta might stabilize the LHDI at large $z$, limiting the $k_{\|}$available for modes driven at the center. This effect, coupled with rising beta and $T_{\mathrm{i}} / T_{\mathrm{e}}$ at the center, could possibly result in the observed rapid drop of LHDI amplitude near the center of the current sheet for the given parameters.

\section{Spatial correlations and propagation characteristics}

The linear theory provides predictions for wavelength and phase velocity of the LHDI, and further evidence for the presence of this instability in MRX could be provided through comparing measured spatial correlations in the fluctuations with the theoretical predictions. In this subsection, studies of the decorrelation length in the measured fluctuations are presented along with statistical dispersion relations derived from the cross-spectrum of two spatially separated differential probes.

Spatial correlations in the fluctuations were investigated using spatially separated double floating Langmuir probes. Three probes were constructed for this purpose, with probeto-probe spacings of $1,3.5$, and $10 \mathrm{~mm}$. The decorrelation length in the fluctuations was investigated through calculating the coherency between separated differential probe signals, which is defined as:

$$
\gamma=\frac{\left|\mathcal{X}_{\mathrm{a}, \mathrm{b}}\right|}{\sqrt{\left|\delta \tilde{\phi}_{\mathrm{f}, \mathrm{a}}\right|^{2}\left|\delta \tilde{\phi}_{\mathrm{f}, \mathrm{b}}\right|^{2}}}
$$

Where $\delta \tilde{\phi}_{\mathrm{f}, \mathrm{a}}$ is the Fourier transform of signal a, and $\mathcal{X}_{\mathrm{a}, \mathrm{b}}=\delta \tilde{\phi}_{\mathrm{f}, \mathrm{a}} \delta \tilde{\phi}_{\mathrm{f}, \mathrm{b}}{ }^{*}$ is the cross spectrum of signals $\mathbf{a}$ and $\mathbf{b}$. Figure 10 shows the mean coherency, averaged over the LHDI feature in the frequency spectrum and over 20 discharges per separation, versus probe separation (normalized to the electron gyroradius). Here the separation, $\Delta x$ is in the toroidal direction, which is the current direction and the expected propagation direction for the LHDI.

The signals are quite coherent at the smallest separation $(1 \mathrm{~mm})$, but the coherency drops rapidly as the separation becomes larger. A decorrelation length for the turbulence can be estimated as the length at which the coherency drops to 1/e. From Figure 10, a decorrelation length $L_{\mathrm{c}} \lesssim 10 \rho_{\mathrm{e}}$ is estimated. This length is comparable to the theoretically predicted wavelength for the strongest growing portion of the LHDI spectrum $\left(\lambda \sim 2 \pi \rho_{\mathrm{e}}\right)$. This 
estimate implies that significant new growth occurs over a single wavelength, an implication which is consistent with the predicted strong linear growth rate for the LHDI $\left(\gamma \sim \omega_{\mathrm{LH}}\right)$.

Dispersion relations of the fluctuations were investigated in the $1 \mathrm{~mm}$ separation case using the statistical method of Beall et al. [53]. Here the local wavenumber at each frequency is calculated from the phase in the cross-spectrum of two spatially separated signals. This computation was performed using two sets of data, one with the probe oriented in the electron diamagnetic direction and the second with the probe oriented in the ion diamagnetic direction. The distinction between these two orientations is made by labeling one of the two probes as primary (call it probe a, for instance), and orienting the two probes such that probe $\mathbf{a}$ is upstream with respect to the second probe in a flow in either the ion or electron diamagnetic direction. This distinction is made primarily as a test for any systematic asymmetries in the probes - if the two probes make measurements in an identical fashion, rotating the probe should result in a positive measurement of $k_{\text {local }}$ in the wave propagation direction and a negative $k_{\text {local }}$ measurement when oriented in the opposite direction. The statistical dispersion relations ( $\omega$ versus local wavenumber) resulting from orientations in the electron (labeled $0^{\circ}$ ) and ion (labeled $180^{\circ}$ ) diamagnetic directions are shown in Figure 11. The gray regions surrounding the black $k_{\perp, \text { local }}$ curves represent the spectral width of the $k_{\perp, \text { local }}$ calculation, which is quite large. The spectral width represents the spread in measured $k_{\perp, \text { local }}$ and the size of this spread is due to the observation of, on average, a large spread in the phase shift in the cross spectrum at each frequency in the turbulence. This fact precludes a statistically significant determination of the wavelength and phase velocity of the fluctuations. However, a preference for propagation in the electron diamagnetic direction is indicated by the measurement of primarily positive local $k_{\perp, \text { local }}$ for orientation in the electron diamagnetic direction, and negative $k_{\perp, \text { local }}$ for the opposite direction. This direction of propagation is consistent with the LHDI when observed in the ion rest frame. Spectroscopic ion flow velocity measurements have been performed in the MRX which suggest that the ion rest frame is the correct lab frame in the current sheet [30].

The large spectral width of these local wavenumber measurements could be attributed to several causes, including deficiencies in the measurement technique and effects in the plasma. One possible deficiency in the measurement technique is the uncertainty in the instantaneous direction of the magnetic field during the measurement. The value of $B_{\mathrm{z}}$ is locally determined (within a few cm using the 1D magnetic probe), however the radial and toroidal fields are 
measured on the other side of the torus (approximately $180^{\circ}$ away in toroidal angle) and may not accurately represent the fields in the toroidal plane of the fluctuation measurement. This may lead to projection effects which would make the wavelength appear longer, but the effect should be proportional to $\cos \theta$ and may not be large enough to explain the observed width. In addition, if there is some $k_{\mathrm{r}}$ to the wave, which we have assumed is zero in the theory presented in Section II, we may be only measuring a projection of $k_{\perp}$ in the toroidal direction. This would lead to a smaller estimate for the wavenumber and a faster apparent phase velocity, consistent with the observations. Nonlinear effects may also contribute to the observed spectral width. The LHDI has a fairly strong predicted linear growth rate in MRX, which is comparable to the real frequency (a prediction supported by decorrelation length estimates). It is therefore not unreasonable to expect rapid nonlinear saturation of the instability and nonlinear modifications to the wavelength spectrum of the turbulence. The linear characteristics of the instability, such as the phase velocity, may not be preserved in the nonlinearly saturated state, and this may be reflected in the measurement.

\section{Comments on the saturated amplitude}

In Section II a brief review of saturation mechanisms for the LHDI was offered, including plateau formation, current relaxation, trapping, electron resonance broadening, and nonlinear mode-mode coupling. Although collisional dissipation is not enough to explain the rate of reconnection in these discharges, sufficient collisions are available such that plateau formation and trapping might not be effective. We will therefore compare the measured amplitude of the fluctuations to the theoretical predicted saturated amplitude due to two models presented in Section II: electron resonance broadening ([28], Eqn. 3) and nonlinear mode-mode coupling ([26], Eqn. 4). The peak amplitude (in both space and time) observed in the radial scan presented Subsection IV A 2 is roughly $\left\langle\delta \phi_{\mathrm{f}}\right\rangle_{\max } \sim 0.4 V$ indicating a normalized potential fluctuation value of $\left(T_{\mathrm{e}} \approx 8 \mathrm{eV}\right)$ :

$$
\frac{e\left\langle\phi_{\mathrm{p}}\right\rangle_{\max }}{T_{\mathrm{e}}} \sim 5 \%
$$

We can now estimate a value for the normalized fluctuating electric field energy density, $\mathcal{E} / n T_{\mathrm{i}}$, where $\mathcal{E} \approx \tilde{E}_{\max }^{2} / 8 \pi=k_{\max }^{2}\left\langle\phi^{2}\right\rangle_{\max } / 8 \pi$. As was discussed in the previous subsection, a statistically significant value for the mode wavelength was not measured, however we can 
estimate the wavenumber in these fluctuations from the linear theory, $k_{\max } \sim \rho_{\mathrm{e}}^{-1}$. Using this estimate, we find that the peak fluctuating electric field value, based on $\phi \sim 0.40 \mathrm{~V}$ and $k \sim \rho_{\mathrm{e}}^{-1} \sim 1700 \mathrm{~m}^{-1}$, is $\tilde{E} \sim 700 \mathrm{~V} / \mathrm{m}$. Using this estimate and $n \sim 2.5 \times 10^{13} \mathrm{~cm}^{-3}$ and $T_{\mathrm{i}} \sim T_{\mathrm{e}} \sim 8 \mathrm{eV}$, we find that in these measurements:

$$
\frac{\mathcal{E}_{\max }}{n T_{\mathrm{i}}} \sim 7 \times 10^{-8}
$$

Now we can compute the predictions, based on measured plasma parameters, of the electron resonance broadening and nonlinear mode coupling saturation models for comparison. The electron resonance broadening model predicts, using $n \sim 2.5 \times 10^{13} \mathrm{~cm}^{-3}, T_{\mathrm{i}} \sim T_{\mathrm{e}} \sim 8$ $\mathrm{eV}, B \sim 100 \mathrm{G}($ at $r \approx 0.36 \mathrm{~cm}):$

$$
\left(\frac{\mathcal{E}}{n T_{\mathrm{i}}}\right) \approx \frac{2}{5} \frac{m_{\mathrm{e}}}{M} \frac{\Omega_{\mathrm{e}}^{2}}{\omega_{\mathrm{p}, \mathrm{e}}^{2}}\left(\frac{T_{\mathrm{i}}}{T_{\mathrm{e}}}\right)^{1 / 4} \frac{V^{2}}{v_{\mathrm{th}, \mathrm{i}}^{2}}=5 \times 10^{-8}
$$

This value is quite comparable to the computed value of $\mathcal{E} / n T_{\mathrm{i}}$ for the measurements reported here $\left(\sim 7 \times 10^{-8}\right)$. However, it should be pointed out that we might expect the electron resonance broadening mechanism to be hampered by electron collisions in MRX, so it might be surprising to find agreement with this prediction. The nonlinear Landau damping saturation mechanism predicts:

$$
\frac{e \phi}{T_{\mathrm{i}}} \approx 2.4\left(\frac{2 m_{\mathrm{e}}}{M}\right)^{1 / 2} \frac{V}{v_{\mathrm{th}, \mathrm{i}}} \approx 20 \%
$$

This prediction is larger than the normalized amplitude deduced from the measurements (5\%), but is quite close considering the limitations of the theoretical model used in this calculation. The theory used to make this estimate ignored coupling of wave energy in unstable long parallel wavelength modes to damped shorter parallel wavelength modes [26]. For this reason, it may overpredict the saturated amplitude in these experiments.

It should be noted that the electron resonance broadening and mode coupling saturation predictions have the same scaling with plasma parameters, and differ only by a constant. This coupled with the fact that both are within a factor of two of the measurement makes it difficult to argue which, if either, is the correct model for saturation of the LHDI in MRX.

\section{Magnetic measurements of LHDI}

A brief discussion of electromagnetic modifications to the LHDI was offered in Section II, suggesting that magnetic fluctuations should be expected along with electrostatic LHDI fluc- 
tuations in high-beta current sheets. While the detailed study performed using electrostatic diagnostics (as presented above) has not yet been reproduced with magnetic diagnostics, initial evidence for electromagnetic LHDI fluctuations have been found. Magnetic pick-up loops were used to study magnetic fluctuations in low-collisionality current sheets $(12 / 10 \mathrm{kV}$, $4 \mathrm{mT}$, hydrogen). These studies revealed high frequency $\left(f \lesssim f_{\mathrm{LH}}\right)$ magnetic fluctuations on the inner edge of the current sheet. The magnetic fluctuations are observed concomitantly with the electrostatic LHDI fluctuations measured using floating probes. These signals are tentatively identified as magnetic LHDI fluctuations, which should be expected to appear due to electromagnetic corrections to the LHDI in high beta current sheets. The amplitude range of these fluctuations is $\delta B \sim 1-10 G$ or $\delta B / B \sim 1-10 \%$, similar to the normalized amplitude in the electrostatic fluctuations. Currently, a detailed study of magnetic fluctuations in MRX is underway which should shed more light on the source of these signals.

\section{B. Role of the LHDI in reconnection in MRX}

One of the primary motivations for studying fluctuations in MRX is to determine the role of any observed fluctuations in the reconnection process. Of particular interest is whether or not the LHDI can generate anomalous resistivity in MRX current sheet or play some other role in establishing the observed enhanced resistivity and fast reconnection rates in MRX [50]. The data and analyses presented in this section suggest that the LHDI is not essential in determining the reconnection rate in MRX. This conclusion stems largely from consideration of the radial profile of the fluctuation amplitude, the time behavior of the fluctuation amplitude and the scaling of the fluctuation amplitude and effective collision rate with Coulomb collisionality.

\section{Radial profiles}

The radial profiles shown in Figure 7 seem to suggest that some penetration of the LHDI into the magnetic null is observed in these measurements. However, It is important to note that the peak of the current density is slightly offset from the magnetic null early in the reconnection process in MRX, due to the asymmetries inherent in the toroidal geometry in MRX. This is demonstrated in Figure 12, where profiles of fitted magnetic field, fit-derived 
current density, and measured fluctuation amplitude are plotted. From this figure, the fluctuation amplitude is not seen to penetrate into the null, consistent with linear theoretical predictions of finite-beta stabilization. The simplest mechanism of anomalous resistivity generation by LHDI turbulence is by effective scattering of the current carrying particles by the wave electric fields. The measured amplitude profile of the LHDI makes it quite difficult to apply this model to the MRX current sheet, as the turbulence is not present at the magnetic null, where it would be needed to provide dissipation.

It is also interesting to discuss the relationship between the current profile and the fluctuation amplitude profile. The current density in MRX is generally observed to be symmetric, while the fluctuation amplitude profile is markedly asymmetric. In addition, the thickness of the current sheet (see, e.g., Fig. 8) seems insensitive to the time history of the amplitude of the LHDI fluctuations. Both of these observations suggest that the fluctuations are not the primary mechanism by which the shape of the current profile is established.

\section{Time behavior of the LHDI amplitude}

An observation which provides further support for a conclusion that the observed fluctuations are not essential for fast reconnection in MRX is the measured time behavior of the fluctuation amplitude. The time behavior of the fluctuation amplitude is compared to that of the average reconnection electric field $\left(E_{\theta}\right)$ and the average central current density $\left(J_{\theta}\right)$ in Figure 13. The reconnection electric field is the time derivative of calculated poloidal flux value in the center of the current sheet, and represents the rate of reconnection (rate of destruction of poloidal flux interior to the current sheet in radius). From this figure, the quasi-steady reconnection phase can be identified as the time period over which the reconnection electric field is steady, roughly from $t=260 \mu \mathrm{s}$ to $t=280 \mu \mathrm{s}$. The fluctuation

amplitude shown in this figure $\left(\left\langle\delta \phi_{\mathrm{f}}\right\rangle_{\max }\right)$ is the peak value in space at each point in time. As was discussed in Section IV A 2, the fluctuation amplitude grows as the current sheet forms and reconnection begins, but is seen to decrease rapidly with time before the end of the quasi-steady reconnection phase (note that the radially integrated or averaged fluctuation power would exhibit an even steeper decline, as is evident when inspecting Fig. 7). Both the reconnection electric field and the peak current density seem rather insensitive to the fairly extreme time behavior of the peak fluctuation amplitude near $t=265 \mu s$. This observation 
suggests that the LHDI fluctuations are not crucial in determining the reconnection rate in MRX, since the reconnection rate is essentially unphased by a rapid change in the amplitude of the fluctuations. In fact, there is some evidence that the reconnection electric field and current density actually increase slightly following the rapid decrease in the fluctuation amplitude near $t=275 \mu \mathrm{s}$. Although this observation is not conclusive, this might suggest that the LHDI actually impedes the reconnection process in MRX. A similar conclusion has been made with respect to recent three-dimensional Hall MHD simulations of reconnection where the LHDI is seen to arise [14].

The measurements reported here are taken only near $z=0$, and it is possible that the fluctuations persist at high amplitude elsewhere in the current sheet even though the amplitude drops dramatically at the measurement location. However, measurements of plasma profiles downstream $(|z|>0)$ have been made, and these measurements suggest shallower density gradients and lower current densities than at $z=0$. Therefore it is expected that the strongest drive for the LHDI should be located at $z=0$. Even if the fluctuations did persist elsewhere, the simplest theoretical picture of anomalous resistivity generation by the LHDI, through effective scattering of the current carrying particles at the null, is unlikely to be valid in light of the observations.

Recent theoretical work has suggested that the LHDI may provide a trigger for reconnection, through either providing an initial resistivity or through nonlinearly steepening the current and density profiles at the edges of a current sheet and triggering additional instabilities such as the drift-kink instability [see, e.g. 13, 42, 54]. It is not clear that there is an onset problem in MRX, as reconnection is driven through boundary perturbations imposed by the external coils (note that this is not unlike the initial tearing mode perturbation imposed on some recent simulations of collisionless reconnection [15]). However, it is possible that the LHDI plays an important role early in the reconnection process in MRX, when its amplitude is strongest, even though it does not seem to influence the eventual quasi-steady reconnection rate. Therefore, future experiments will investigate the influence of the LHDI on the onset of reconnection in MRX current sheets. 


\section{Scaling of fluctuation amplitude and quasilinear resistivity with collisionality}

The discussions already presented in this section provide evidence supporting the argument that the measured fluctuations are not of crucial importance in setting the reconnection rate in MRX. An additional data set which provides further support for this conclusion was was taken to explore the dependence of the fluctuation amplitude and computed quasilinear resistivity on the collisionality in MRX current sheets. Collisionality in MRX current sheets is characterized by the parameter $\lambda_{\mathrm{mfp}} / \delta$, where $\delta$ is the width of the current sheet and $\lambda_{\mathrm{mfp}}$ is the electron mean free path against Coulomb collisions. As the collisionality is lowered in MRX current sheets, the measured toroidal reconnection electric field, $E_{\theta}$, is no longer balanced by classical collisional drag at the center of the current sheet, $E_{\theta} / \eta_{\mathrm{sp}} j_{\theta} \gg 1$ (where $\eta_{\mathrm{sp}}$ is the classical Spitzer perpendicular resistivity) [49]. The size of this discrepancy, which could be characterized as a resistivity enhancement, increases rapidly with decreasing collisionality. If the measured LHDI fluctuations were responsible for this measured resistivity enhancement, one might expect a strong dependence of the LHDI amplitude and effective collision rate on the Coulomb collisionality in MRX.

Fig. 14(a) shows the measured peak fluctuation amplitude (peak amplitude in both space and time) versus $\lambda_{\operatorname{mfp}} / \delta$ from a scan of fill pressure. The amplitude of the fluctuations does tend to increase with decreasing collisionality (increasing $\lambda_{\mathrm{mfp}} / \delta$ ). However, if the fluctuation amplitude is normalized to the measured electron temperature, which from Boltzmann's equation might be considered as an estimate of $\delta n / n$ in the turbulence, we find that there is essentially no change in this quantity with collisionality, as shown in Fig. 14(b).

Theoretical estimates of effective collision rates produced by LHDI fluctuations depend on the normalized amplitude of the fluctuations, $\mathcal{E}_{\mathrm{k}} / n T \sim(\delta n / n)^{2}$ (see Eqn. 8). Figure 14(b) then suggests that the effective collision rate provided by the LHDI fluctuations in MRX should be fairly constant as the collisionality is drastically changed in the current sheet. However, as the collisionality is raised in MRX $\nu_{\mathrm{e}, \mathrm{i}}$ increases dramatically, and therefore the normalized LHDI resistivity, $\nu_{\mathrm{LHDI}} / \nu_{\mathrm{e}, \mathrm{i}}$ might behave in a manner consistent with the observed resistivity enhancement in MRX. We can now compute the normalized effective LHDI collision rate for this set of data, using Eqn. 8 along with the measured amplitude, plasma parameters and the linear theoretical estimates for the LHDI shown in Figure 9. For 
example, for the lowest collisionality data point in Figure 14,

$$
\begin{aligned}
\nu_{\mathrm{LHDI}} & =\operatorname{Im}\left(k_{\perp} \frac{4 \omega_{\mathrm{p}, \mathrm{i}}^{2}}{k_{\perp}^{2} v_{\mathrm{th}, \mathrm{i}}^{2}} \zeta_{\mathrm{i}} \mathrm{Z}\left(\zeta_{\mathrm{i}}\right)\right)_{k_{\perp, \max }} \frac{T_{\mathrm{i}}}{m_{\mathrm{e}} V} \frac{\mathcal{E}}{n T_{\mathrm{i}}} \\
& =\frac{\omega_{\mathrm{p}, \mathrm{i}}^{2}}{\Omega_{\mathrm{i}}^{2}} \frac{v_{\mathrm{th}, \mathrm{i}}}{V} \frac{\mathcal{E}}{n T_{\mathrm{i}}} \operatorname{Im}\left(\frac{4}{k_{\perp} \rho_{\mathrm{e}}} \zeta_{\mathrm{i}} \mathrm{Z}\left(\zeta_{\mathrm{i}}\right)\right)_{k_{\perp, \max }} \omega_{\mathrm{LH}} \\
& \approx 0.6 \omega_{\mathrm{LH}}=26 \mathrm{MHz}
\end{aligned}
$$

This estimate is actually lower than the Coulomb collision rate for that data point, $\nu_{\mathrm{e}, \mathrm{i}} \approx$ $35 \mathrm{MHz}$, suggesting a resistivity enhancement of less than a factor of two. Figure 15 shows the computed LHDI resistivity enhancement along with the measured resistivity enhancement $\left(E / \eta_{\mathrm{sp}} j\right)$ as a function of collisionality for all the data points in the pressure scan. While the LHDI resistivity enhancement does increase with decreasing collisionality, it is clearly insufficient to explain the observed value of $E / \eta_{\mathrm{sp}} j$. It should be noted that the effective collision rate is computed using the maximum fluctuation amplitude (maximum in both time and space), and therefore provides a very generous estimate of the LHDI resistivity. The amplitude at the null point, where $E / \eta_{\mathrm{sp}} j$ is measured, is significantly lower than this peak amplitude, and an estimate of the effective collisionality there should be more than an order of magnitude lower. It should also be noted that the theory used in computing the effective LHDI collisionality is a collisionless theory, and it is not clear how these estimates change when the Coulomb collisionality is close to the linear frequency of the instability, as is the case here.

\section{SUMMARY AND DISCUSSION}

In this paper, detailed measurements of floating potential fluctuations in the MRX current sheet were presented. These measurements have led to the first experimental identification of the lower-hybrid drift instability in a laboratory current sheet, and to the first opportunity for a detailed study of the role of this instability in magnetic reconnection. Support for identifying the measured potential fluctuations as being due to the LHDI was provided by detailed measurements of the frequency spectrum, radial amplitude profiles and spatial correlations. A local linear theory of the LHDI was used to successfully explain asymmetries observed in the measured radial fluctuation amplitude profile. Correlation measurements indicated a decorrelation length in the turbulence which was comparable to the theoretically 
predicted wavelength of the LHDI, an observation which is consistent with a theoretically predicted strong peak linear growth rate. Measurements of phase velocity in the fluctuations suggested a preference for propagation in the electron diamagnetic direction, but a statistically significant value for the phase velocity was not found due to a large variations in the measured phase at each frequency in the turbulence. Estimates of the expected saturation amplitude by electron resonance broadening and nonlinear mode coupling were made based on measured plasma parameters. The estimate for the measured potential fluctuation amplitude was found to be comparable to the electron resonance broadening estimate, but roughly a factor of 4 lower than the nonlinear Landau damping estimate.

The observations presented suggest that the measured potential fluctuations do not play an essential role in determining the quasi-steady reconnection rate in MRX. The role of the LHDI in the reconnection process in MRX was explored through studying the spatial and temporal behavior of the fluctuation amplitude and through studying the dependence of the fluctuation amplitude on the current sheet collisionality. The observed radial profile of the fluctuations is consistent with several theoretical predictions that the LHDI should not penetrate to the high-beta null point. The fluctuation amplitude was observed to drop dramatically during reconnection while the reconnection rate (electric field) was steady. The mechanism for the drop in amplitude is still not fully understood, but this observation makes it difficult to claim that the LHDI is providing anomalous dissipation during reconnection in MRX. Finally, a study of the effect of collisionality in the current sheet on the fluctuation amplitude and computed effective collisionality was performed. The normalized fluctuation amplitude was found to be fairly insensitive to the collisionality in MRX current sheets. The quasilinear estimate of the LHDI collisionality was found to fall short of the Coulomb collision rate in low collisionality discharges, even when the peak fluctuation amplitude is used in the computation, further suggesting that the fluctuations are not responsible for enhancing the resistivity in MRX current sheets.

Magnetic fluctuations have also been observed in MRX, and any relationship between these fluctuations and the reconnection rate is currently the subject of intense investigations. While fluctuation studies will continue, with current data it is difficult to construct a theory of fast reconnection in MRX based solely on extending MHD with an anomalous resistivity generated by the observed electrostatic potential fluctuations. Therefore future experimental campaigns on MRX will also focus on exploring alternative fast reconnection 
mechanisms, including looking for signatures of fast reconnection mediated by the Hall term in the generalized Ohm's law. There is some consistency between the data and the Halldominated models in the observation of a current sheet thickness proportional to the ion skin depth; simulations have shown that an ion current layer can exist at this scale [16]. However, the simulations predict that the current sheet can have two scales, and that an inner, electron current scale can be as small as $c / \omega_{\mathrm{p}, \mathrm{e}}$ (depending on the source of dissipation). Such a current layer could solve the electron force balance problem in MRX through an increased (and currently unresolved) current density, $j^{*}$, such that $E=\eta j^{*}$ (dissipation could be provided by collisions). The real experimental tests of these simulations will therefore only come through attempting to resolve smaller scale features in the MRX current sheet - features that are potentially many times smaller than the current size of individual magnetic detectors in MRX. The development of new diagnostics for this purpose is already underway.

\section{ACKNOWLEDGMENTS}

The authors would like to thank D. Cylinder and R. Cutler for their excellent technical support. MRX is jointly funded by DOE (contract number DE-AC02-76CH03073), NASA, and NSF. T.A.C. acknowledges support from NSF Graduate Research and NASA GSRP Fellowships.

\section{APPENDIX A: DERIVATION OF LHDI ELECTRON DENSITY PERTURBA-} TION

In the following we use the electrostatic approximation and introduce $k_{\perp}=\sqrt{k_{x}^{2}+k_{y}^{2}}$ and $k_{\|}=k_{z}$. The Vlasov equation is used to calculate perturbed distribution functions, from which the perturbed charge densities are calculated and used in Poisson's equation.

$$
\left(\frac{d \delta f_{\mathrm{e}}}{d t}\right)_{0}=-\frac{q}{m} \mathbf{E} \cdot \frac{\partial f_{\mathrm{e}}^{0}}{\partial \mathbf{v}}
$$

We use the method of characteristics to solve for $\delta f_{\mathrm{e}}$, integrating along the zero-order orbits of the particles: 


$$
\begin{aligned}
\delta f_{\mathrm{e}} & =-\frac{q}{m} \int_{-\infty}^{t} d t^{\prime}\left(\mathbf{E} \cdot \frac{\partial}{\partial \mathbf{v}} f_{\mathrm{e}}^{0}\right)_{v^{\prime}, r^{\prime}, t^{\prime}} \\
& =\frac{q}{m} \int_{-\infty}^{t} d t^{\prime}\left[-\mathbf{v} \cdot \nabla \phi \frac{2 F_{\mathrm{m}, \mathrm{e}}}{v_{\mathrm{th}, \mathrm{e}}^{2}}+\frac{\epsilon_{\mathrm{n}}}{\Omega_{\mathrm{e}}} F_{\mathrm{m}, \mathrm{e}}(\nabla \phi)_{y}\right] \\
& =-\frac{2 q}{m} \frac{F_{\mathrm{m}, \mathrm{e}}}{v_{\mathrm{th}, \mathrm{e}}^{2}} \phi_{t^{\prime}=t}+\frac{2 q}{m} \frac{F_{\mathrm{m}, \mathrm{e}}}{v_{\mathrm{th}, \mathrm{e}}^{2}} \int_{-\infty}^{t} d t^{\prime} \frac{\partial \phi}{\partial t^{\prime}}+\frac{i k_{y} q \epsilon_{\mathrm{n}}}{m \Omega_{\mathrm{e}}} F_{\mathrm{m}, \mathrm{e}} \int_{-\infty}^{t} d t^{\prime} \phi
\end{aligned}
$$

The final step is accomplished by using $\mathbf{v} \cdot \nabla \phi=d \phi / d t-\partial \phi / \partial t$, and by assuming $\phi_{t^{\prime} \rightarrow-\infty}=0$.

In order to complete the time integrals, we must first solve the single particle equations of motion for the electrons. These are:

$$
\begin{gathered}
\frac{d \mathbf{v}}{d t}=\frac{q}{m_{\mathrm{e}}} \frac{\mathbf{v} \times \mathbf{B}(x)}{c} \\
\frac{d \mathbf{r}}{d t}=\mathbf{v}
\end{gathered}
$$

Assuming that the gradient scale length in the magnetic field is much longer than the electron gyroradius, we can use the guiding center expansion to obtain the electron orbit. Introducing the variable $\tau=t^{\prime}-t$, we find:

$$
\begin{aligned}
y^{\prime} & \approx \frac{v_{\perp}}{\Omega_{\mathrm{e}}} \cos \left(\varphi+\Omega_{\mathrm{e}} \tau\right)-\frac{v_{\perp}}{\Omega_{\mathrm{e}}} \cos \varphi-\frac{1}{2} \epsilon_{\mathrm{b}} \frac{v_{\perp}^{2}}{\Omega_{\mathrm{e}}} \tau \\
x^{\prime} & \approx \frac{v_{\perp}}{\Omega_{\mathrm{e}}} \sin \left(\varphi+\Omega_{\mathrm{e}} \tau\right)-\frac{v_{\perp}}{\Omega_{\mathrm{e}}} \sin \varphi \\
z^{\prime} & =-v_{\|} \tau
\end{aligned}
$$

Where $\epsilon_{\mathrm{b}}=(1 / B) \partial B / \partial x$ and $\epsilon_{\mathrm{b}} v_{\perp}^{2} / 2 \Omega_{\mathrm{e}}=V_{\nabla \mathrm{B}}$, the electron $\nabla B$ drift speed. Here we are ignoring oscillating terms of order $\epsilon_{\mathrm{b}} v_{\perp}^{2} / \Omega_{\mathrm{e}}$. If we assume $\phi=\tilde{\phi} \exp (i \mathbf{k} \cdot \mathbf{r}-i \omega t)$, then the equation for $\delta f_{\mathrm{e}}$ becomes:

$$
\begin{aligned}
\delta f_{\mathrm{e}}= & -\frac{2 q \tilde{\phi}}{m} \frac{F_{\mathrm{m}, \mathrm{e}}}{v_{\mathrm{th}, \mathrm{e}}^{2}}\left[1+i\left(\omega-k_{y} v_{\mathrm{D}, \mathrm{e}}\right) \times\right. \\
& \left.\int_{0}^{\infty} d \tau \exp \left(-i\left(\frac{k_{\perp} v_{\perp}}{\Omega_{\mathrm{e}}}\left(\cos \left(\varphi+\Omega_{\mathrm{e}} \tau\right)-\cos \varphi\right)\right)+\left(\omega-k_{\|} v_{\|}-k_{y} V_{\nabla \mathrm{B}}\right) \tau\right)\right]
\end{aligned}
$$

Using the fact that

$$
\exp (i z \sin \varphi)=\sum_{n=-\infty}^{\infty} \exp (i n \varphi) J_{n}(z)
$$


and

$$
\exp \left(i z \sin \left(\varphi+\Omega_{\mathrm{e}} \tau\right)\right)=\sum_{n=-\infty}^{\infty} \exp \left(i m\left(\varphi+\Omega_{\mathrm{e}} \tau\right)\right) J_{n}(z)
$$

$\delta f_{\mathrm{e}}$ becomes:

$$
\begin{aligned}
\delta f_{\mathrm{e}}= & -\frac{2 q}{m} \frac{F_{\mathrm{m}, \mathrm{e}}}{v_{\mathrm{th}, \mathrm{e}}^{2}} \tilde{\phi}\left[1-\left(\omega-k_{y} v_{\mathrm{D}, \mathrm{e}}\right) \times\right. \\
& \left.\sum_{n, m}\left(\frac{J_{n}(z) J_{m}(z) \exp (i(m-n)(\varphi-\pi / 2))}{\omega-k_{\|} v_{\|}-k_{y} V_{\nabla \mathrm{B}}-m \Omega_{\mathrm{e}}}\right)\right]
\end{aligned}
$$

Where we have introduced the electron diamagnetic velocity, $v_{\mathrm{D}, \mathrm{e}}=\epsilon_{\mathrm{n}} v_{\mathrm{th}, \mathrm{e}}^{2} / 2 \Omega_{\mathrm{e}}$ and $z=$ $k_{\perp} v_{\perp} / \Omega_{\mathrm{e}}$. The perturbed electron density can now be calculated by integrating $\delta f_{\mathrm{e}}$ over velocity space:

$$
\begin{aligned}
\delta n_{\mathrm{e}}= & -\frac{2 q n_{\mathrm{o}}}{m v_{\mathrm{th}, \mathrm{e}}^{2}} \tilde{\phi}-\frac{2 q}{m v_{\mathrm{th}, \mathrm{e}}^{2}} \tilde{\phi}\left(\omega-k_{y} v_{\mathrm{D}, \mathrm{e}}\right) \times \\
& \sum_{m, n} \int v_{\perp} d v_{\perp} \int d v_{\|} \frac{J_{n}(z) J_{m}(z) F_{\mathrm{m}, \mathrm{e}}}{\omega-k_{\|} v_{\|}-k_{y} V_{\nabla \mathrm{B}}-m \Omega_{\mathrm{e}}} \int d \varphi \exp (i(m-n)(\varphi-\pi / 2))
\end{aligned}
$$

The velocity phase integral is nonzero only for $m=n$. The integral over $v_{\|}$evaluates to a plasma dispersion function, $Z$. The perturbed electron density then becomes:

$$
\begin{aligned}
\delta n_{\mathrm{e}}= & -\frac{2 q n_{\mathrm{o}}}{m v_{\mathrm{th}, \mathrm{e}}^{2}} \tilde{\phi}-\frac{2 q n_{\mathrm{o}}}{m v_{\mathrm{th}, \mathrm{e}}^{2}} \tilde{\phi}\left(\omega-k_{y} v_{\mathrm{D}, \mathrm{e}}\right) \frac{2}{k_{\|} v_{\mathrm{th}, \mathrm{e}}} \times \\
& \sum_{n} \int x d x \exp \left(-x^{2}\right) J_{n}^{2}\left(k_{\perp} \rho_{\mathrm{e}} x\right) \mathrm{Z}\left(\frac{\omega-k_{y} \bar{V}_{\nabla \mathrm{B}} x^{2}-n \Omega_{\mathrm{e}}}{k_{\|} v_{\mathrm{th}, \mathrm{e}}}\right)
\end{aligned}
$$

Where we have introduced $x=v_{\perp} / v_{\mathrm{th}, \mathrm{e}}$, and $\bar{V}_{\nabla \mathrm{B}}=\epsilon_{\mathrm{b}} v_{\mathrm{th}, \mathrm{e}}^{2} / 2 \Omega_{\mathrm{e}}$. The frequency range of interest for the LHDI is $\omega \sim \omega_{\mathrm{LH}} \ll \Omega_{\mathrm{e}}$, and we will therefore keep only the $n=0$ term in the sum. So the final expression for the perturbed electron density is then:

$$
\delta n_{\mathrm{e}}=-\frac{2 q n_{\mathrm{o}}}{m v_{\mathrm{th}, \mathrm{e}}^{2}} \tilde{\phi}-\frac{2 q n_{\mathrm{o}}}{m v_{\mathrm{th}, \mathrm{e}}^{2}} \tilde{\phi}\left(\omega-k_{y} v_{\mathrm{D}, \mathrm{e}}\right) \frac{2}{k_{\|} v_{\mathrm{th}, \mathrm{e}}} \int x d x \exp \left(-x^{2}\right) J_{0}^{2}\left(k_{\perp} \rho_{\mathrm{e}} x\right) \mathrm{Z}\left(\frac{\omega-k_{y} \bar{V}_{\nabla \mathrm{B}} x^{2}}{k_{\|} v_{\mathrm{th}, \mathrm{e}}}\right)
$$


[1] D. Biskamp, Phys. Rep. 237, 179 (1994).

[2] S. Syrovatskii, Sov. Phys. JETP 33, 933 (1971).

[3] E. Parker, J. Geophys. Res. 62, 509 (1957).

[4] S. Syrovatskii, in Solar terrestrial physics, edited by E. Dyer (Reidel, Dordrecht, 1972), p. 119.

[5] M. Ugai, Phys. Plasmas 2, 388 (1995).

[6] H. Petschek, in Physics of Solar Flares (1963), p. 425.

[7] J. Heyvaerts, E. Priest, and D. Rust, Ap. J. 216, 123 (1977).

[8] G. Haerendel, J. Atmos. Terr. Phys. 40, 343 (1978).

[9] F. Coroniti and A. Evitar, Ap. J. Suppl. Series 33, 89 (1977).

[10] J. Huba, N. Gladd, and K. Papadopoulos, Geophys. Res. Lett. 4, 125 (1977).

[11] I. Shinohara, T. Nagai, M. Fujimoto, T. Terasawa, T. Mukai, K. Tsuruda, and T. Yamamoto, J. Geophys. Res. 103, 20365 (1998).

[12] R. Davidson, N. Gladd, C. Wu, and J. Huba, Phys. Fluids 20, 301 (1977).

[13] R. Horiuchi and T. Sato, Phys. Plasmas 6, 4565 (1999).

[14] B. Rogers, J. Drake, and M. Shay, Geophys. Res. Lett. 27, 3157 (2000).

[15] J. Birn, J. Drake, M. Shay, B. Rogers, R. Denton, M. Hesse, M. Kuznetsova, Z. Ma, A. Bhattachargee, A. Otto, et al., J. Geophys. Res. Space. Phys. 106, 3715 (2001).

[16] M. Shay, J. Drake, R. Denton, and D. Biskamp, J. Geophys. Res. 103, 9165 (1998).

[17] M. Yamada, H. Ji, S. Hsu, T. Carter, R. Kulsrud, Y. Ono, and F. Perkins, Phys. Rev. Lett. 78, 3117 (1997).

[18] T. A. Carter, H. Ji, F. Trintchouk, M. Yamada, and R. M. Kulsrud, Phys. Rev. Lett. 88, 015001 (2002).

[19] T. A. Carter, Ph.D. thesis, Princeton University (2001).

[20] N. Krall and P. Liewer, Phys. Rev. A 4, 2094 (1971).

[21] P. Yoon, A. Lui, and C. Chang, Phys. Plasmas 1, 3033 (1994).

[22] N. Krall and M. Rosenbluth, Phys. Fluids 5, 1435 (1962).

[23] J. Huba and C. Wu, Phys. Fluids 19, 988 (1976).

[24] M. Yamada, H. Ji, S. Hsu, T. Carter, R. Kulsrud, and F. Trintchouk, Phys. Plasmas 7, 1781 
(2000).

[25] T. Stix, Waves in Plasmas (American Institute of Physics, New York, 1992).

[26] J. Drake, P. Guzdar, A. Hassam, and J. Huba, Phys. Fluids 27, 1148 (1984).

[27] R. Davidson and N. Gladd, Phys. Fluids 18, 1327 (1975).

[28] J. Huba, N. Gladd, and K. Papadopoulos, J. Geophys. Res. 83, 5217 (1978).

[29] R. Davidson, Phys. Fluids 21, 1376 (1978).

[30] S. Hsu, T. Carter, G. Fiksel, H. Ji, R. Kulsrud, and M. Yamada, Phys. Plasmas 8, 1916 (2001).

[31] D. Winske and P. Liewer, Phys. Fluids 21, 1017 (1978).

[32] J. Huba and K. Papadopoulos, Phys. Fluids 21, 121 (1978).

[33] S. Gary, Phys. Fluids 23, 1193 (1980).

[34] J. Brackbill, D. Forslund, K. Quest, and D. Winske, Phys. Fluids 27, 2682 (1984).

[35] D. Winske and D. Hewett, Phys. Rev. Lett. 35, 937 (1975).

[36] Y. Chen and C. Birdsall, Phys. Fluids 26, 180 (1983).

[37] M. Tanaka and T. Sato, J. Geophys. Res. 86, 5541 (1981).

[38] Z. Zhu and R. Winglee, J. Geophys. Res. 101, 4885 (1996).

[39] K. Yamanaka, Phys. Scripta 17, 15 (1977).

[40] D. Winske, Phys. Fluids 24, 1069 (1981).

[41] W. Daughton, Phys. Plasmas 6, 1329 (1999).

[42] W. Daughton, Phys. Plasmas (2002), in press.

[43] D. Gurnett, L. Frank, and R. Lepping, J. Geophys. Res. 81, 6059 (1976).

[44] H. Fahrbach, W. Köppendörfer, M. Münich, J. Neuhauser, H. Röhr, G. Schramm, J. Sommer, and E. Holzhauer, Nucl. Fusion 21, 257 (1981).

[45] E. Choueiri, A. Kelly, and R. Jahn, in Proceedings of the 22nd International Electric Propulsion Conference (CentroSpazio, Viareggio, Italy, 1991), pp. IEPC 91-100, copies can be ordered from CentroSpazio, Via A. Ghirerdesca, 5, 26014 Ospedallto, Pisa, Italy.

[46] D. Tilley, E. Choueiri, A. Kelly, and R. Jahn, J. Propul. Power 12, 381 (1996).

[47] E. Harris, Il Nuovu Cimento 23, 115 (1962).

[48] S.-L. Chen and T. Sekiguchi, J. Appl. Phys. 36, 2363 (1965).

[49] H. Ji, M. Yamada, S. Hsu, R. Kulsrud, T. Carter, and S. Zaharia, Phys. Plasmas 6, 1743 (1999). 
[50] H. Ji, M. Yamada, S. Hsu, and R. Kulsrud, Phys. Rev. Lett. 80, 3256 (1998).

[51] S. Hsu, G. Fiksel, T. Carter, H. Ji, R. Kulsrud, and M. Yamada, Phys. Rev. Lett. 84, 3859 (2000).

[52] W. Gekelman and R. Stenzel, J. Geophys. Res. 89, 2715 (1984).

[53] J. Beall, Y. Kim, and E. Powers, J. Appl. Phys. 53, 933 (1982).

[54] P. Yoon and A. Lui, Phys. Fluids B 5, 1993 (1993). 
FIG. 1: Real frequency and growth rates for the LHDI using parameters relevant to the MRX experiment.

FIG. 2: Peak growth rate for the LHDI as a function of beta.

FIG. 3: Schematic of the MRX device, including representative current sheet location and fluctuation measurement geometry.

FIG. 4: Traces of plasma current and measured floating potential signal along with an FFT of the signal. Current sheet formation and reconnection occur roughly from $t=240 \mu s$ to $280 \mu s$.

FIG. 5: (a) Average floating potential power spectrum in hydrogen at different average field strengths. (b) Frequency at peak fluctuation amplitude in hydrogen versus magnetic field.

FIG. 6: Average fluctuation spectra in hydrogen and helium for similar magnetic field values.

FIG. 7: Radial profiles of RMS fluctuation amplitude at $z=0$ and current density in the MRX current sheet at four times.

FIG. 8: Contours of RMS fluctuation amplitude in the $r-t$ plane. Superimposed is the trajectory of the current sheet center $\left(R_{\mathrm{o}}\right)$ and the current sheet thickness $\left(R_{\mathrm{o}} \pm \delta\right)$.

FIG. 9: (a) Radial profiles of fitted average magnetic field and density, including a smooth fit to the density profile (two-temperature Lorentzian). (b) Electron beta calculated from measured electron density, electron temperature, and magnetic field. (c) Fluctuation amplitude and current density profiles at $t=264 \mu s$. (d) Computed peak growth rate profile for the LHDI, for the measured profiles and for $T_{\mathrm{i}} / T_{\mathrm{e}}=1,2,3$. 
FIG. 10: The mean coherency of spatially separated measurements of LHDI fluctuations in MRX.

FIG. 11: Statistical dispersion relations for two probe orientations $\left(0^{\circ} / 180^{\circ}=\right.$ electron/ion diamagnetic direction).

FIG. 12: Profiles of magnetic field, current density, and fluctuation amplitude at $t=264 \mu s$, demonstrating that no significant penetration of the LHDI into the magnetic null is observed.

FIG. 13: Time traces of reconnection electric field, peak current density, and peak RMS fluctuation amplitude in $12 / 10 \mathrm{kV} 4 \mathrm{mT}$ hydrogen discharges.

FIG. 14: (a) Fluctuation amplitude and (b) normalized fluctuation amplitude versus collisionality from a scan in fill pressure.

FIG. 15: Measured resistivity enhancement and computed LHDI resistivity enhancement as a function of collisionality 


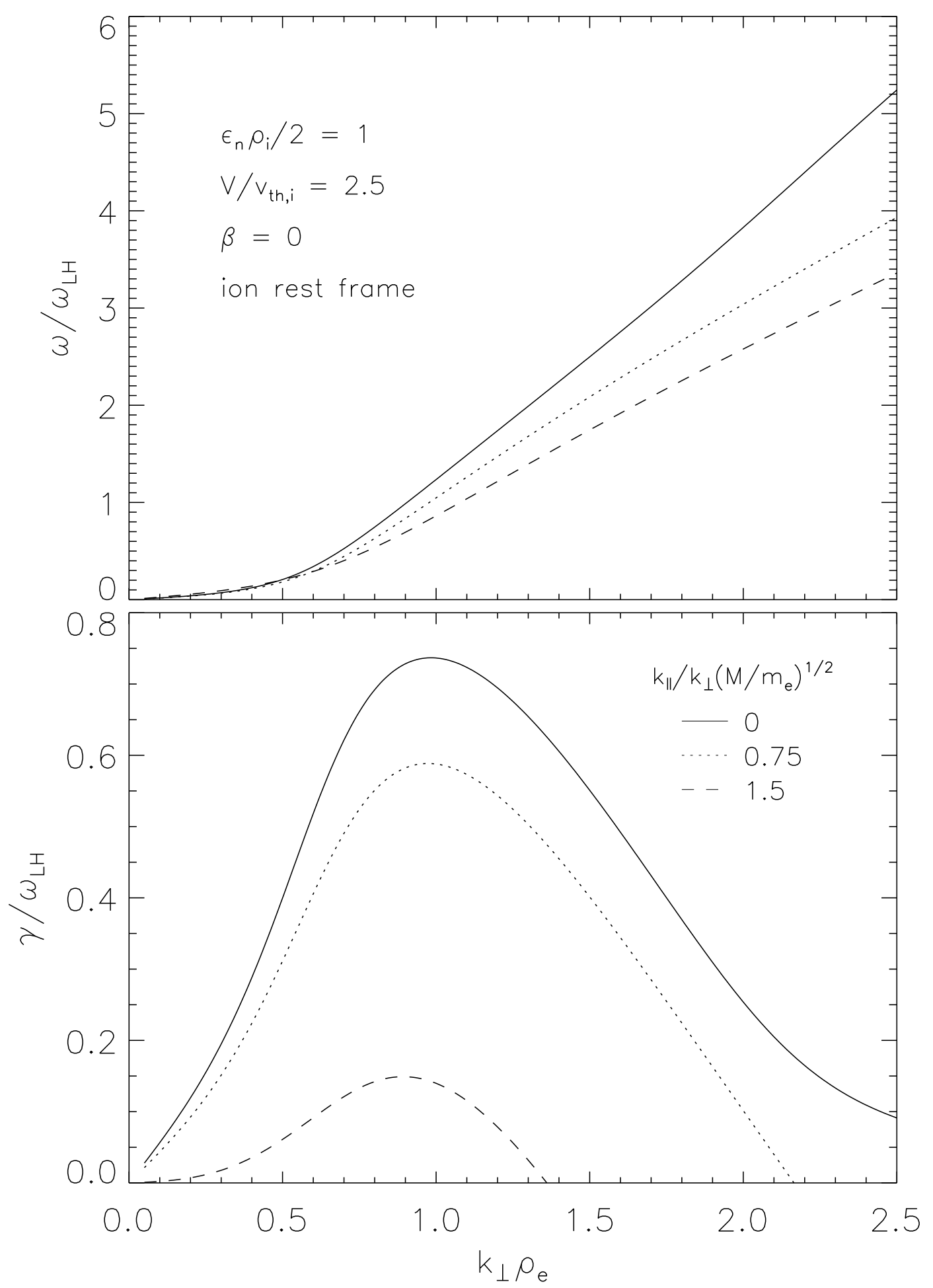

Fig 1 


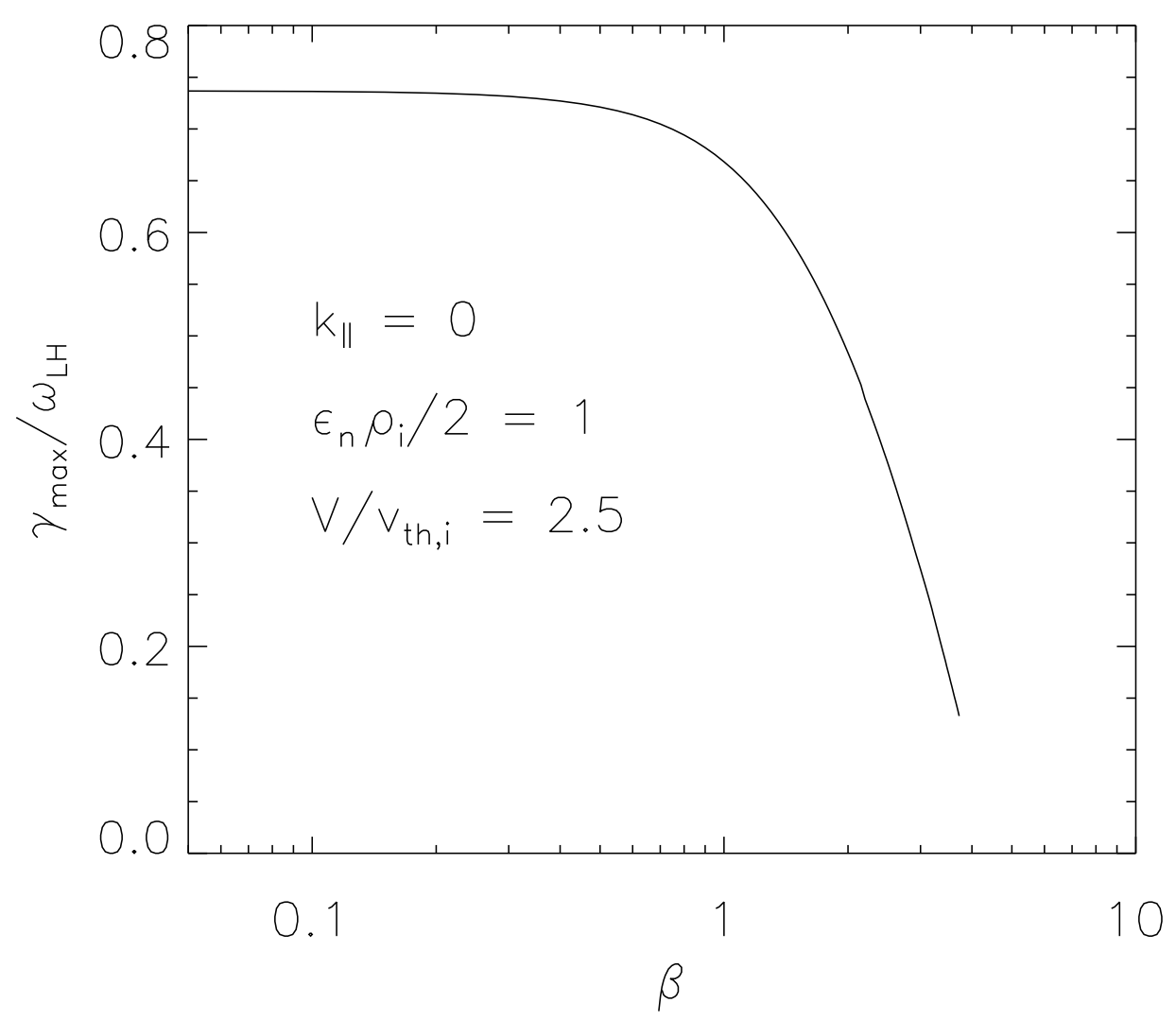

Fig 2 


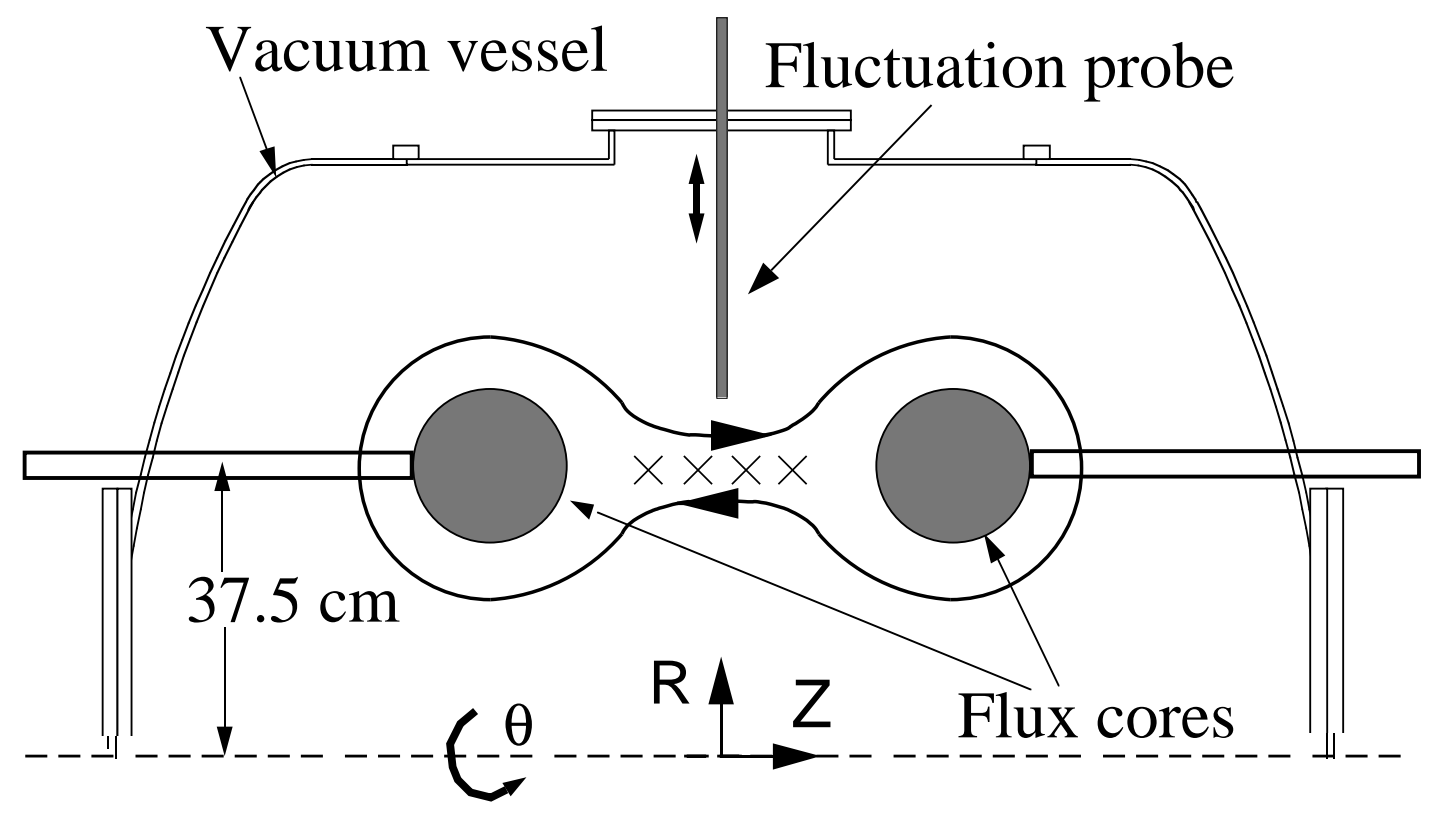

Fig 3 


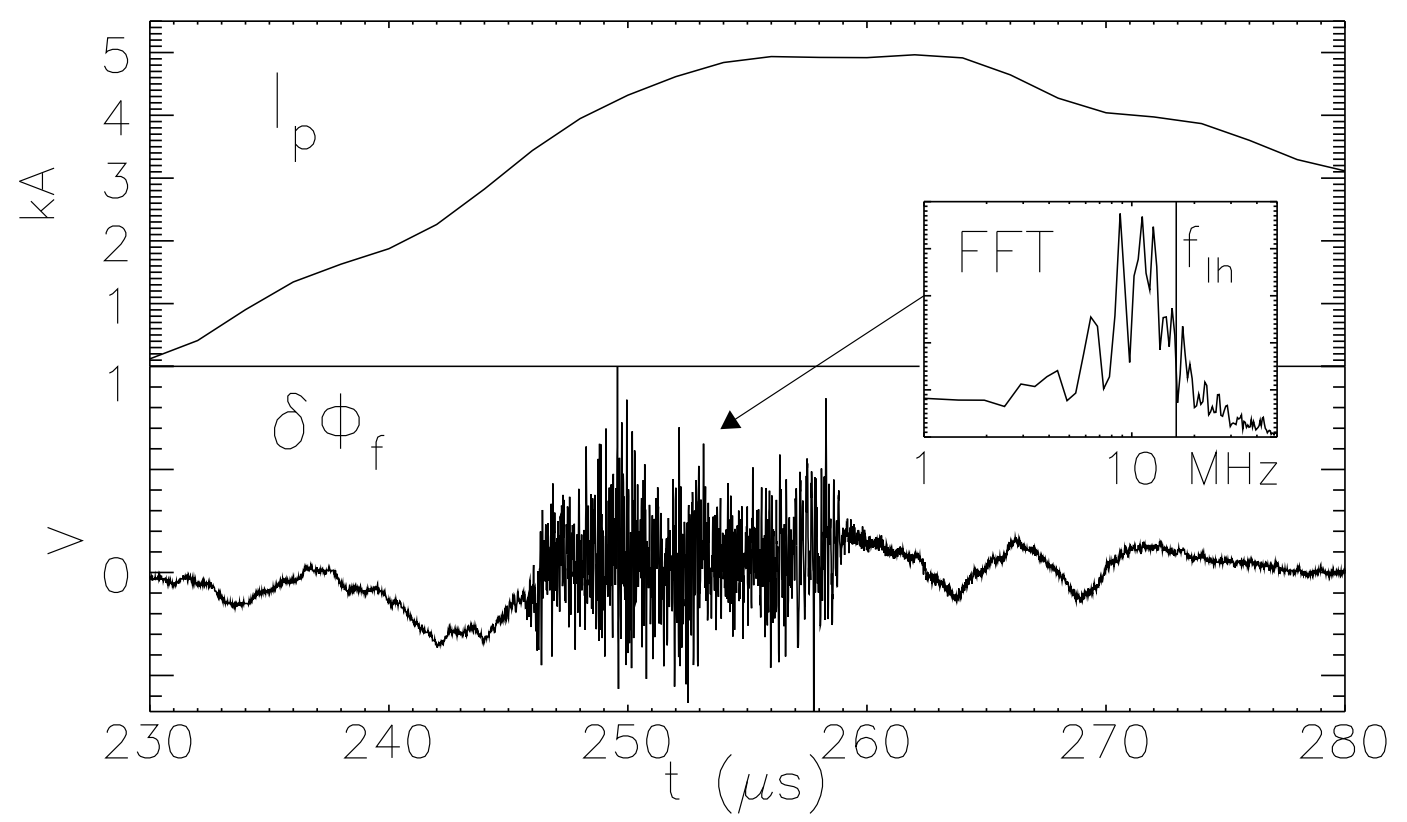

Fig 4 


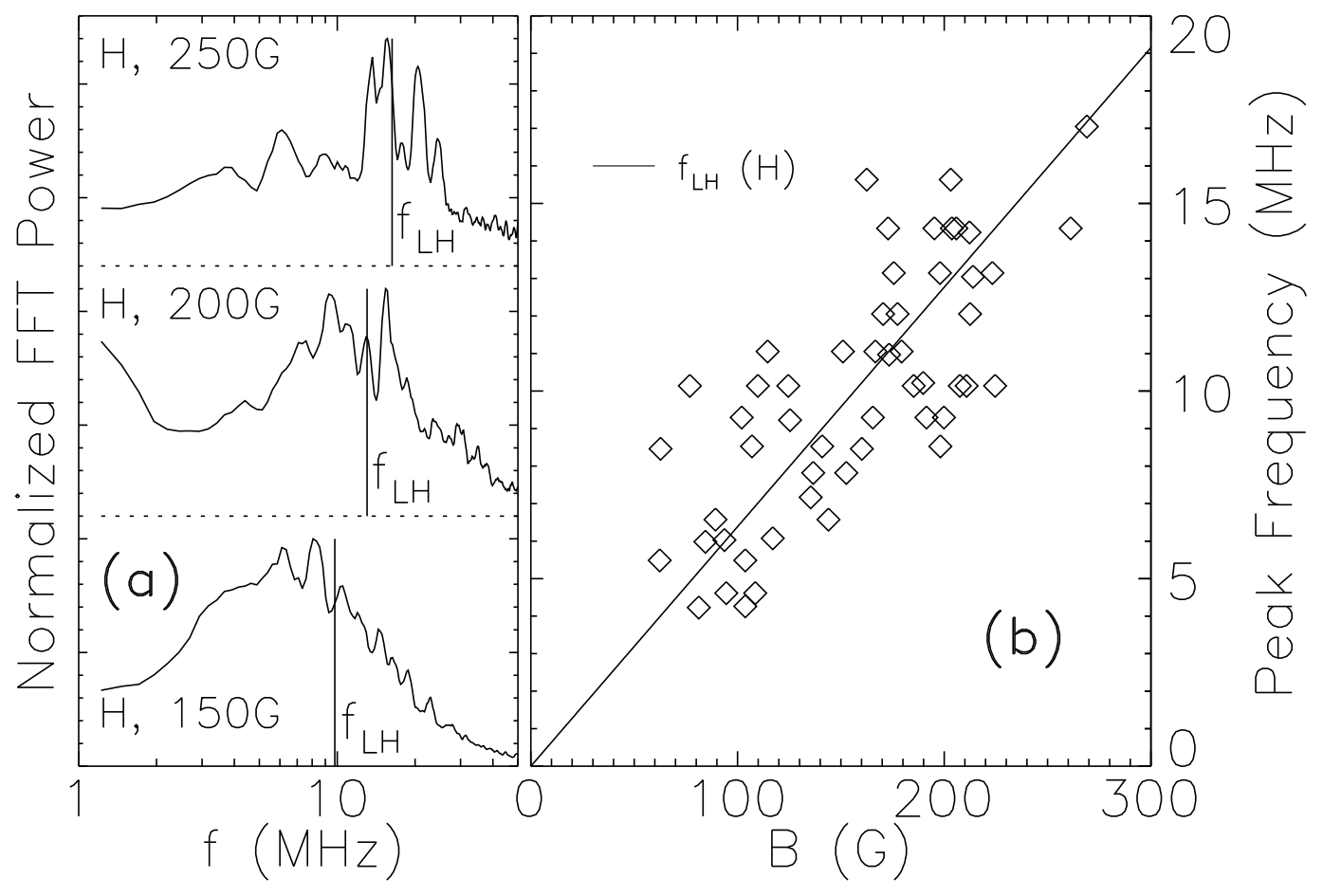

Fig 5 


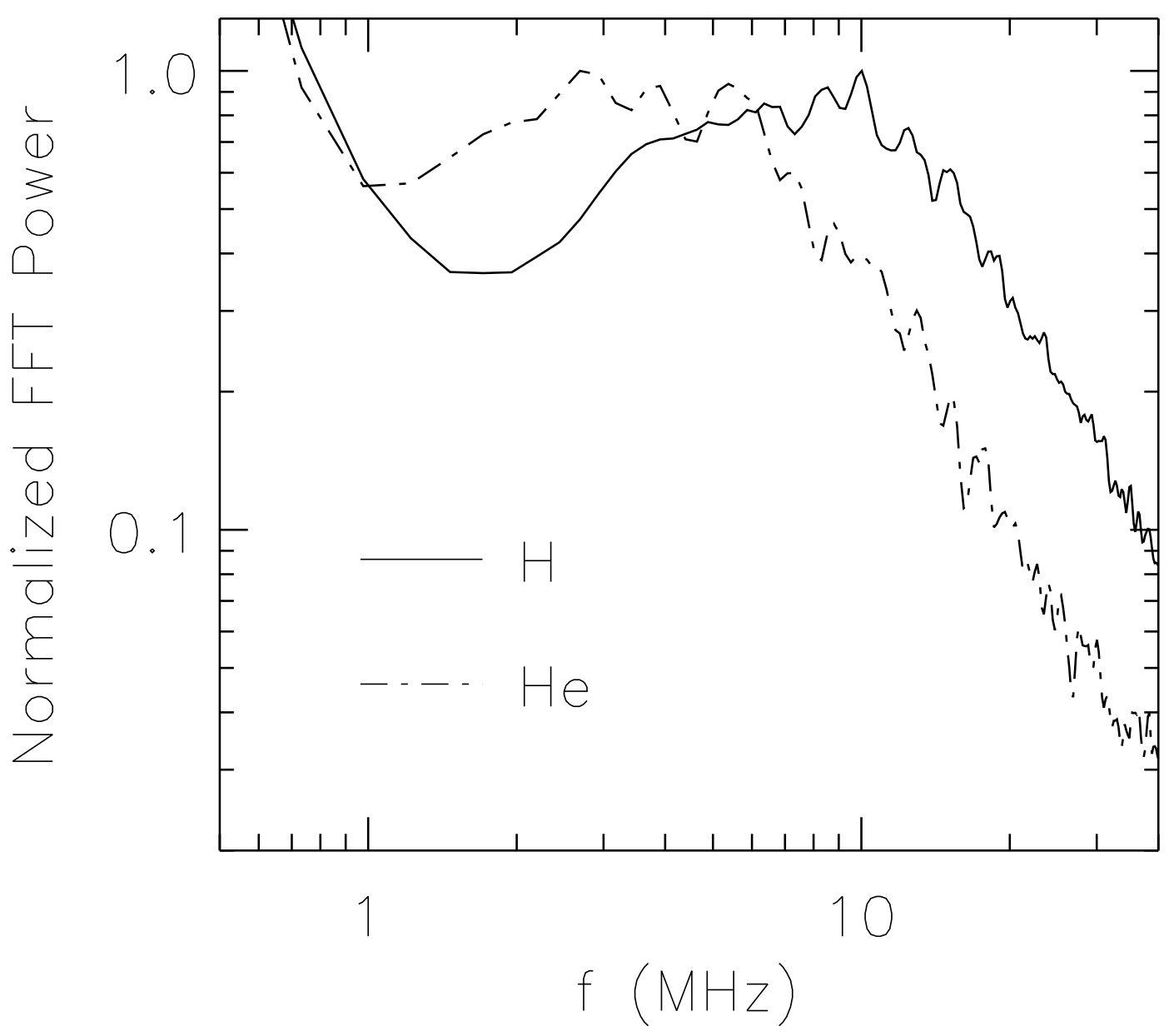

Fig 6 


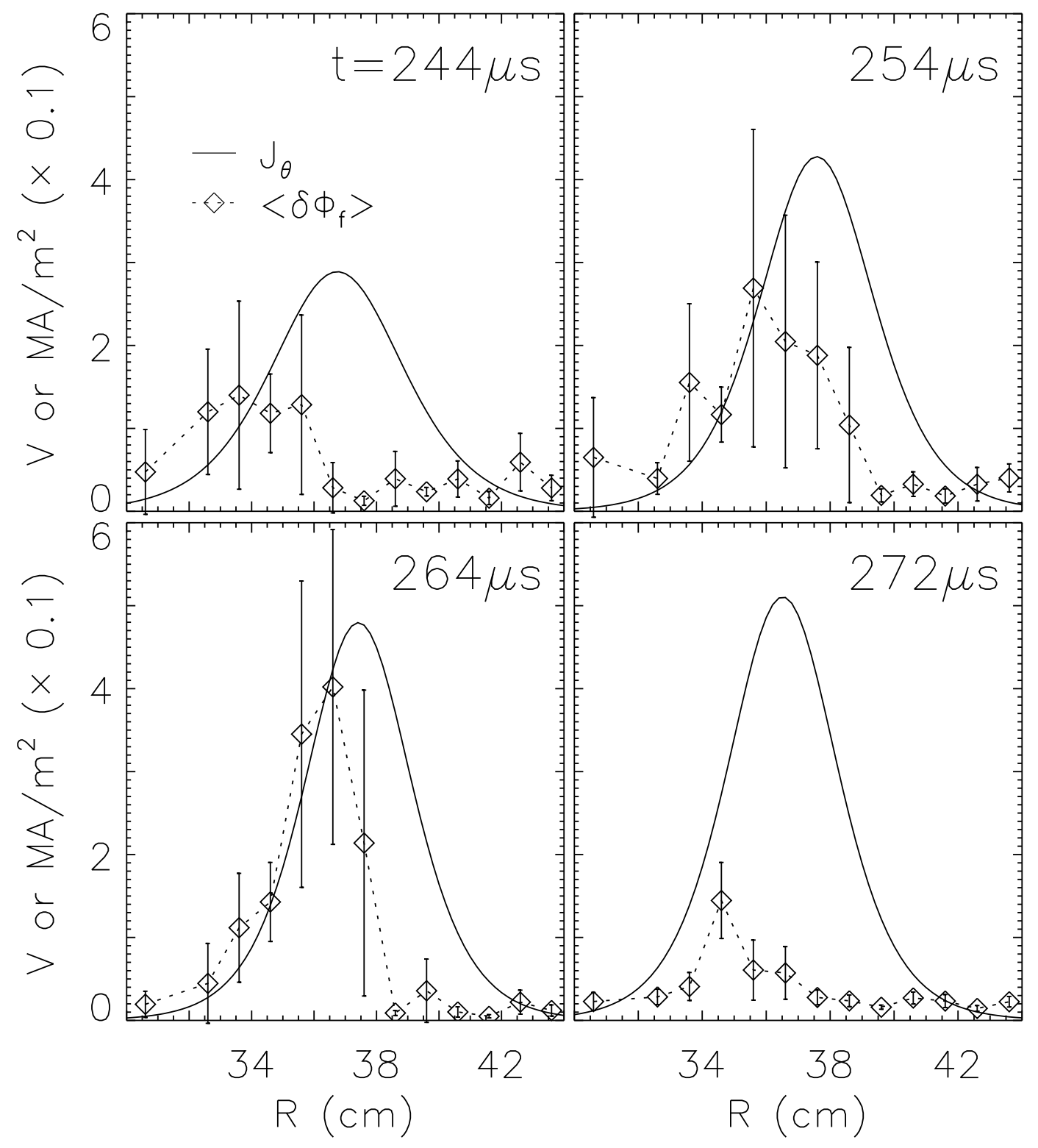

Fig 7 


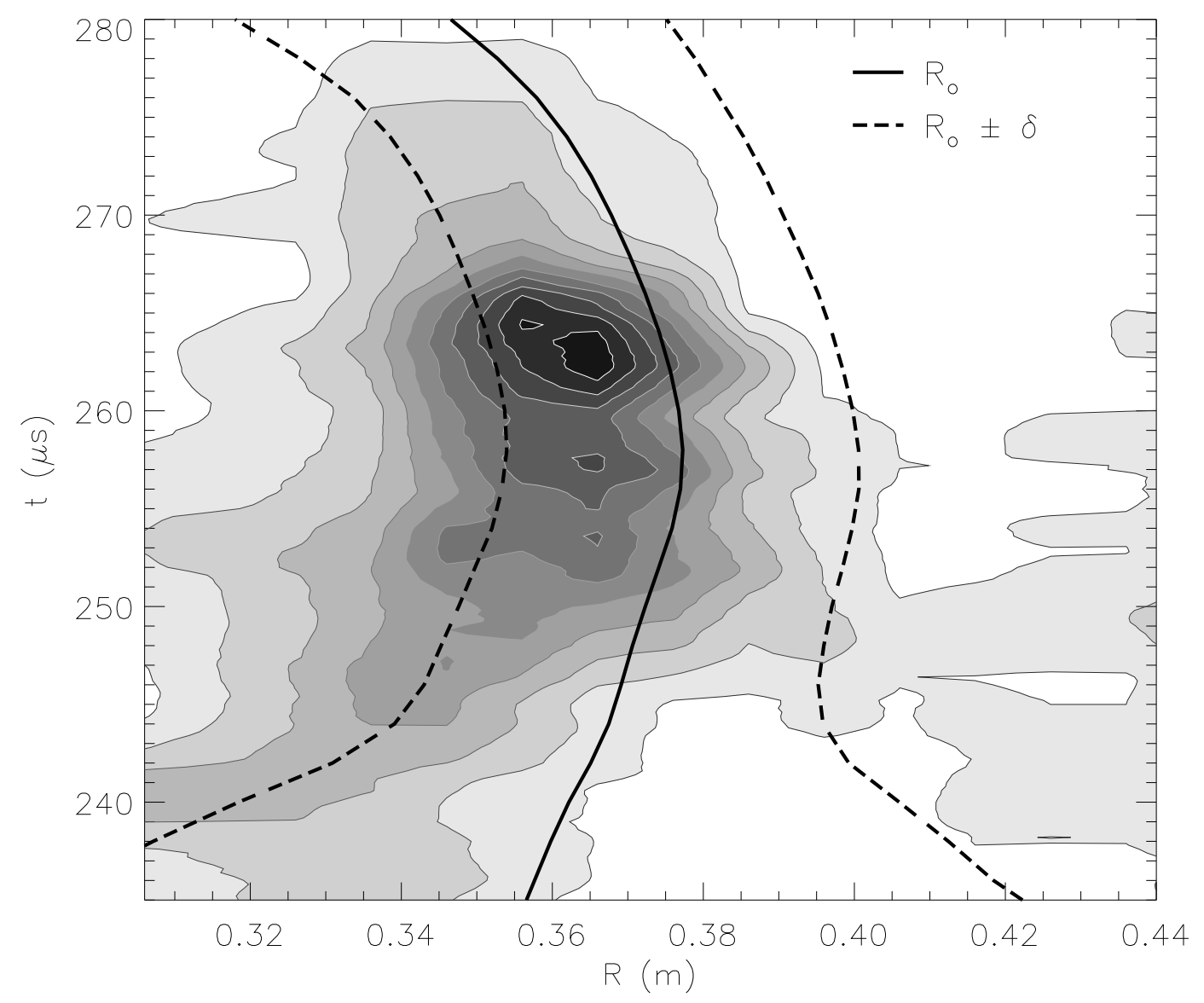

Fig 8 

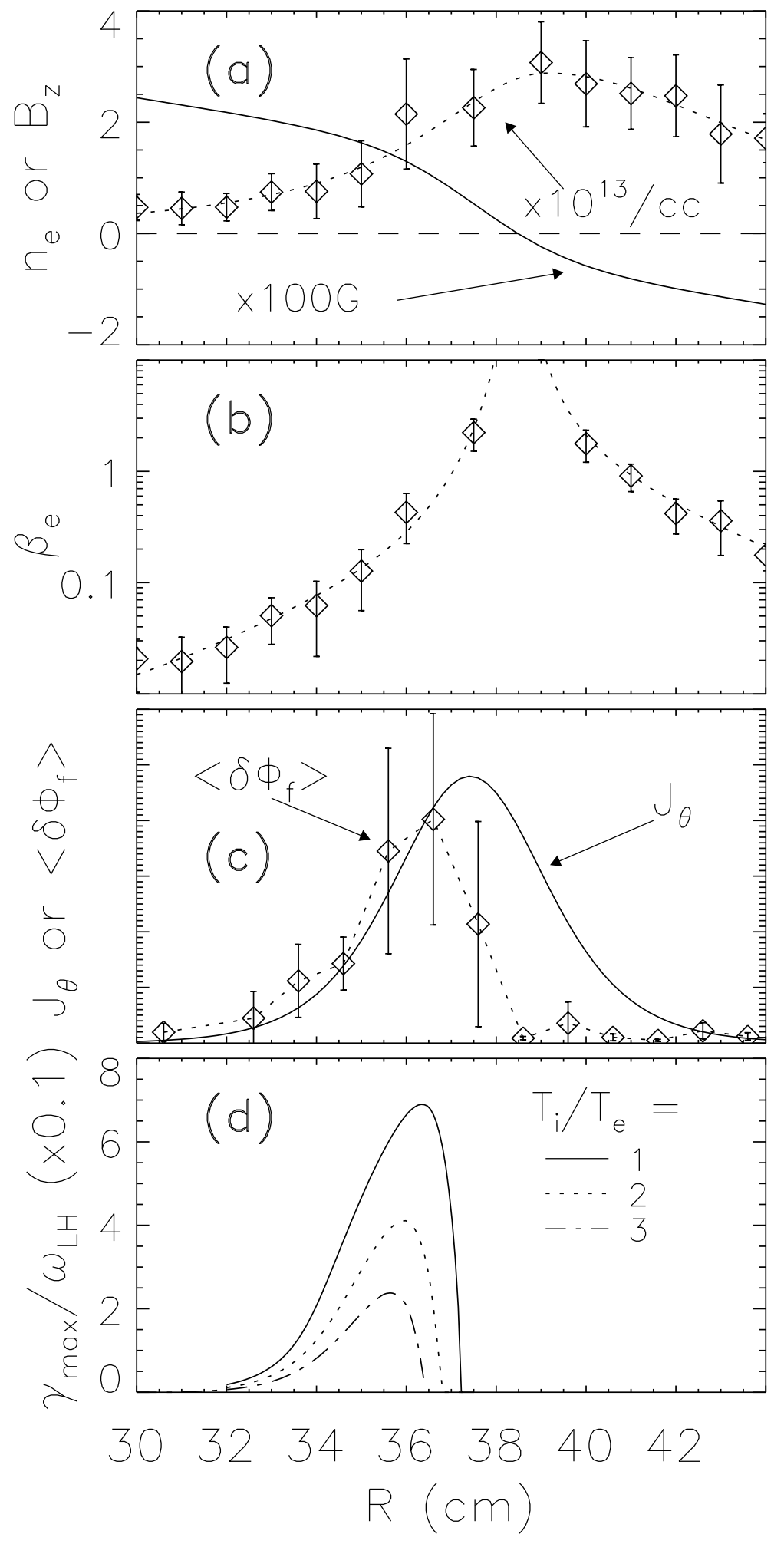

Fig 9 


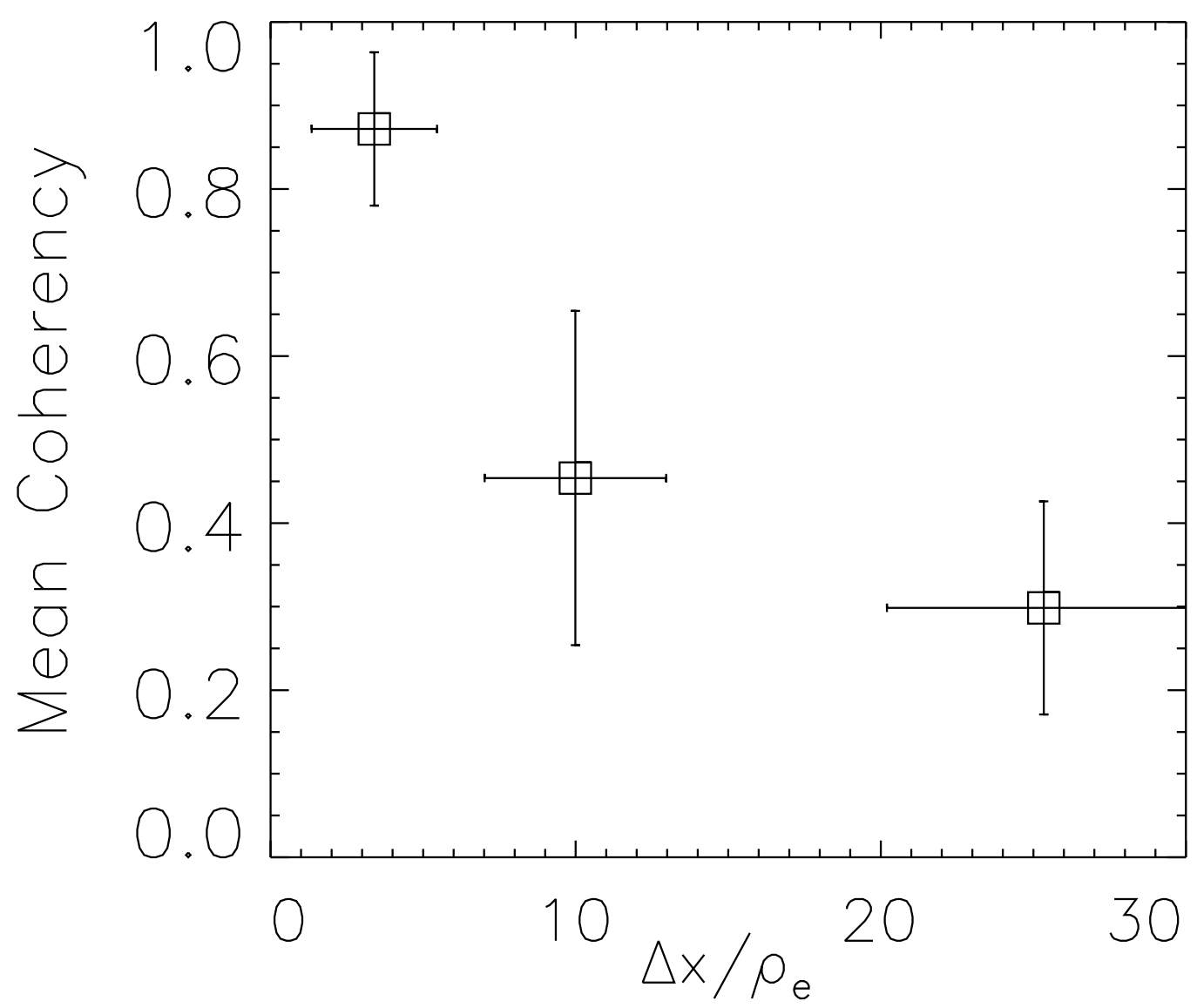

Fig 10 


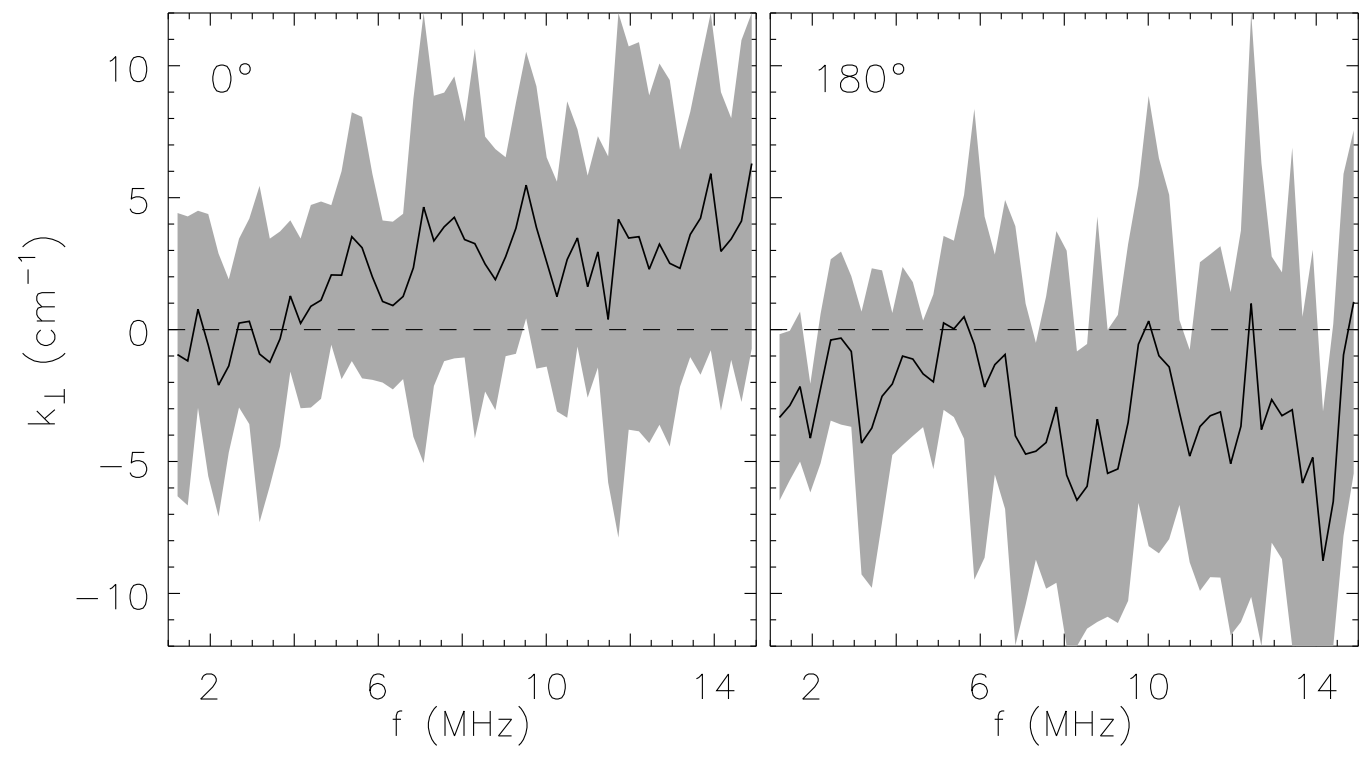

Fig 11 


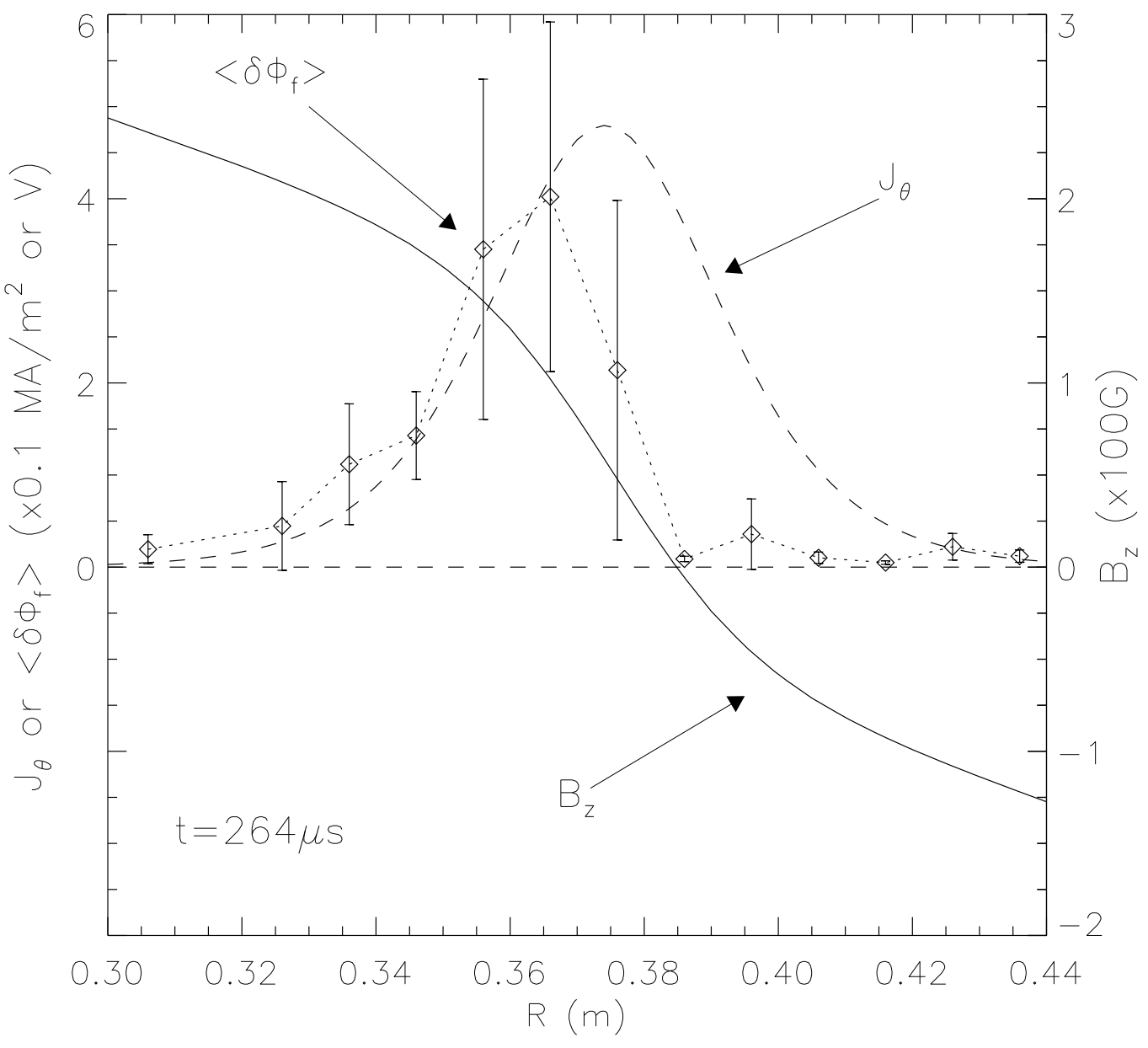

Fig 12 


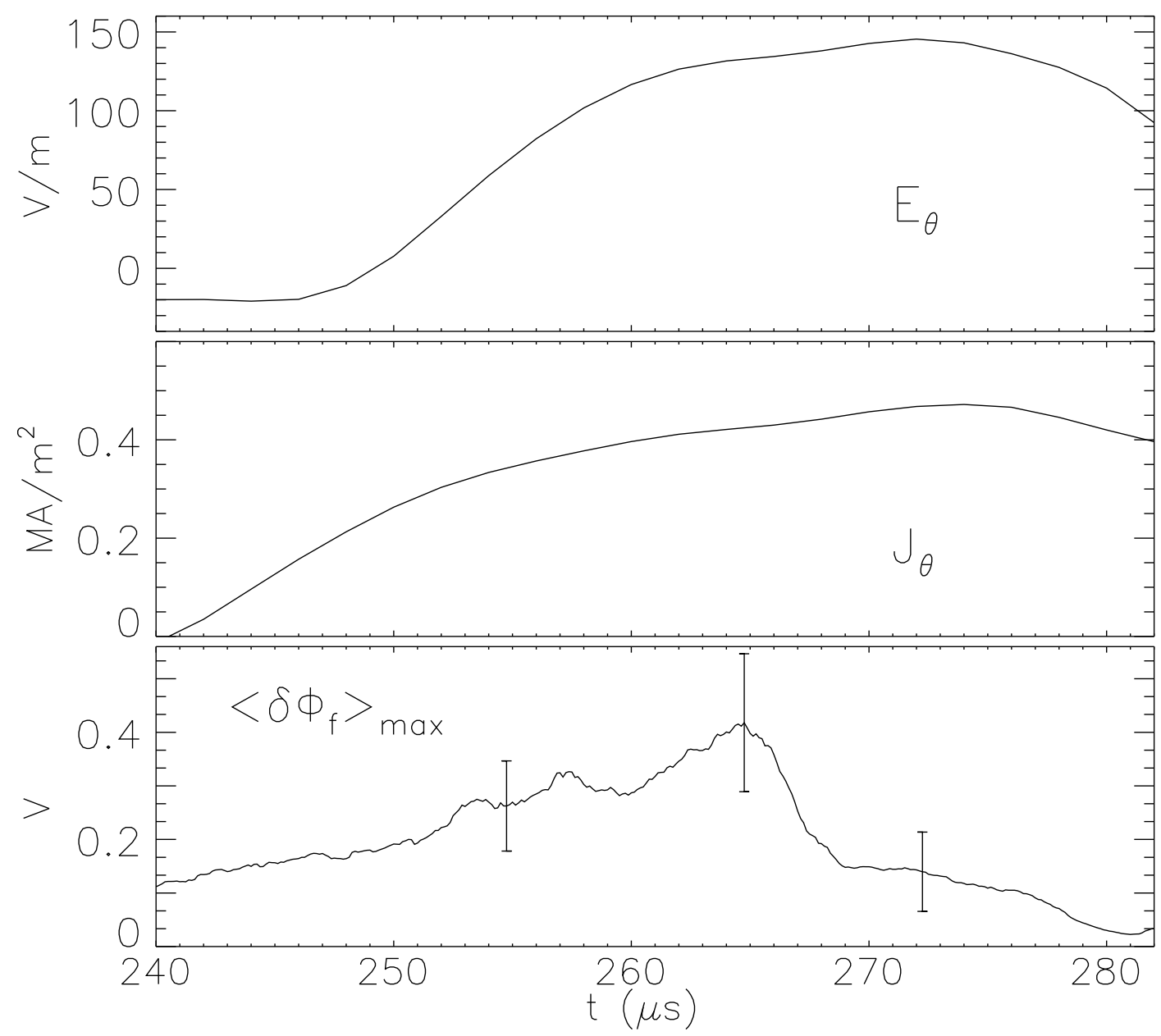

Fig 13 


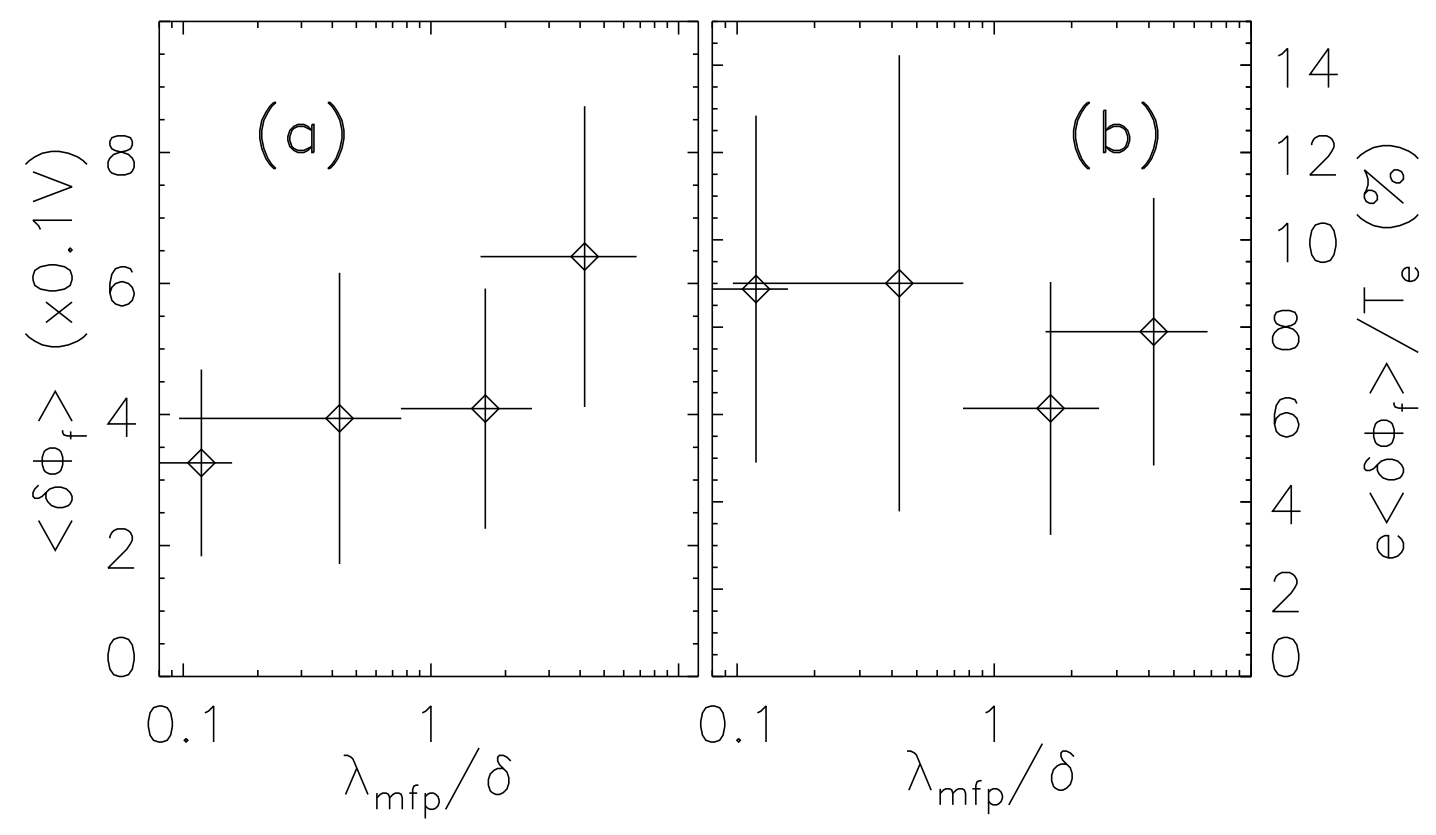

Fig 14 


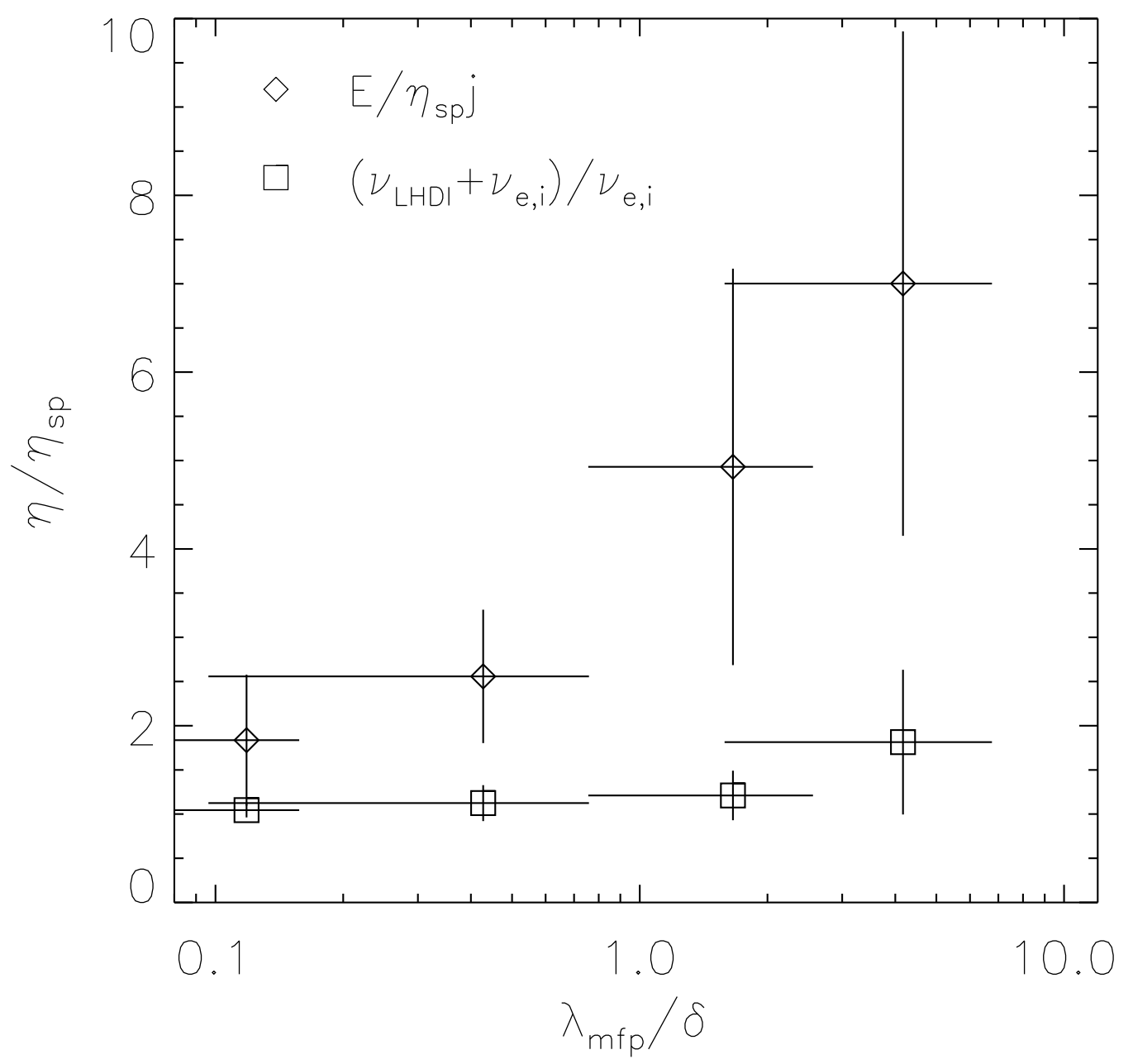

Fig 15 


\section{External Distribution}

Plasma Research Laboratory, Australian National University, Australia

Professor I.R. J ones, Flinders University, Australia

Professor J oão Canalle, Instituto de Fisica DEQ/IF - UERJ , Brazil

Mr. Gerson O. Ludwig, Instituto Nacional de Pesquisas, Brazil

Dr. P.H. Sakanaka, Instituto Fisica, Brazil

The Librarian, Culham Laboratory, England

Library, R61, Rutherford Appleton Laboratory, England

Mrs. S.A. Hutchinson, JET Library, England

Professor M.N. Bussac, Ecole Polytechnique, France

Librarian, Max-Planck-Institut für Plasmaphysik, Germany

J olan Moldvai, Reports Library, MTA KFKI-ATKI, Hungary

Dr. P. Kaw, Institute for Plasma Research, India

Ms. P.J . Pathak, Librarian, Insitute for Plasma Research, India

Ms. Clelia De Palo, Associazione EURATOM-ENEA, I taly

Dr. G. Grosso, Instituto di Fisica del Plasma, Italy

Librarian, Naka Fusion Research Establishment, J AERI, J apan

Library, Plasma Physics Laboratory, Kyoto University, J apan

Research Information Center, National Institute for Fusion Science, J apan

Dr. O. Mitarai, Kyushu Tokai University, J apan

Library, Academia Sinica, Institute of Plasma Physics, People's Republic of China

Shih-Tung Tsai, Institute of Physics, Chinese Academy of Sciences, People's Republic of China

Dr. S. Mirnov, TRINITI, Troitsk, Russian Federation, Russia

Dr. V.S. Strelkov, Kurchatov Institute, Russian Federation, Russia

Professor Peter Lukac, Katedra Fyziky Plazmy MFF UK, Mlynska dolina F-2, Komenskeho Univerzita, SK-842 15 Bratislava, Slovakia

Dr. G.S. Lee, Korea Basic Science Institute, South Korea

Mr. Dennis Bruggink, Fusion Library, University of Wisconsin, USA

Institute for Plasma Research, University of Maryland, USA

Librarian, Fusion Energy Division, Oak Ridge National Laboratory, USA

Librarian, Institute of Fusion Studies, University of Texas, USA

Librarian, Magnetic Fusion Program, Lawrence Livermore National Laboratory, USA

Library, General Atomics, USA

Plasma Physics Group, Fusion Energy Research Program, University of California at San Diego, USA

Plasma Physics Library, Columbia University, USA

Alkesh Punjabi, Center for Fusion Research and Training, Hampton University, USA

Dr. W.M. Stacey, Fusion Research Center, Georgia Institute of Technology, USA

Dr. J ohn Willis, U.S. Department of Energy, Office of Fusion Energy Sciences, USA

Mr. Paul H. Wright, Indianapolis, Indiana, USA 
The Princeton Plasma Physics Laboratory is operated by Princeton University under contract with the U.S. Department of Energy.

\author{
Information Services \\ Princeton Plasma Physics Laboratory \\ P.O. Box 451 \\ Princeton, NJ 08543
}

Phone: 609-243-2750

Fax: 609-243-2751

e-mail: pppl_info@pppl.gov

Internet Address: http://www.pppl.gov 